\title{
Relationship between Transport Anisotropy and Nematicity in FeSe
}

\author{
Jack M. Bartlett, ${ }^{1,2, *}$ Alexander Steppke, ${ }^{1, *},{ }^{*}$ Suguru Hosoi@ $\odot,{ }^{3,4}$ Hilary Noad $\odot,{ }^{1}$ Joonbum Park, ${ }^{1}$ Carsten Timm, ${ }^{5,6}$ \\ Takasada Shibauchi® ${ }^{3}$ Andrew P. Mackenzie $\oplus^{1,2}$ and Clifford W. Hicks ${ }^{1,7, \$}$ \\ ${ }^{1}$ Max Planck Institute for Chemical Physics of Solids, Nöthnitzer Str 40, 01187 Dresden, Germany \\ ${ }^{2}$ SUPA, School of Physics and Astronomy, University of St. Andrews, \\ St. Andrews KY16 9SS, United Kingdom \\ ${ }^{3}$ Department of Advanced Materials Science, University of Tokyo, Kashiwa, Chiba 277-8561, Japan \\ ${ }^{4}$ Department of Materials Engineering Science, Osaka University, Toyonaka, Osaka 560-8531, Japan \\ ${ }^{5}$ Institute of Theoretical Physics, Technische Universität Dresden, 01062 Dresden, Germany \\ ${ }^{6}$ Würzburg-Dresden Cluster of Excellence ct.qmat, Technische Universität Dresden, \\ 01062 Dresden, Germany \\ ${ }^{7}$ School of Physics and Astronomy, University of Birmingham, Birmingham B15 2TT, United Kingdom
}

(Received 21 July 2020; revised 9 January 2021; accepted 9 February 2021; published 19 May 2021)

The mechanism behind the nematicity of FeSe is not known. Through elastoresitivity measurements it has been shown to be an electronic instability. However, thus far measurements have extended only to small strains, where the response is linear. Here, we apply large elastic strains to FeSe and perform two types of measurement. (1) Using applied strain to control twinning, the nematic resistive anisotropy at temperatures below the nematic transition temperature $T_{s}$ is determined. (2) Resistive anisotropy is measured as nematicity is induced through applied strain at fixed temperature above $T_{s}$. In both cases, as nematicity strengthens, the resistive anisotropy peaks at about $7 \%$, then decreases. Below $\approx 40 \mathrm{~K}$, the nematic resistive anisotropy changes sign. We discuss possible implications of this behavior for theories of nematicity. In addition, we report the following. (1) Under experimentally accessible conditions with bulk crystals, stress, rather than strain, is the conjugate field to the nematicity of FeSe. (2) At low temperatures the twin boundary resistance is $\sim 10 \%$ of the sample resistance, and must be properly subtracted to extract intrinsic resistivities. (3) Biaxial in-plane compression increases both in-plane resistivity and the superconducting critical temperature $T_{c}$, consistent with a strong role of the $y z$ orbital in the electronic correlations.

DOI: 10.1103/PhysRevX.11.021038

\section{INTRODUCTION}

At an electronic-nematic transition, electronic interactions drive a spontaneous reduction in rotational symmetry without introducing translational or time-reversal symmetry breaking. Electronic nematicity affects all the Fermi surfaces of a metal, and therefore its fluctuations can have powerful effects $[1,2]$. It is potentially an integral part of the high-temperature superconductivity of iron-based

\footnotetext{
*These authors contributed equally to this work.

† steppke@cpfs.mpg.de

${ }^{\ddagger}$ C.Hicks.1@bham.ac.uk
}

Published by the American Physical Society under the terms of the Creative Commons Attribution 4.0 International license. Further distribution of this work must maintain attribution to the author(s) and the published article's title, journal citation, and DOI. Open access publication funded by the Max Planck Society.
Subject Areas: Condensed Matter Physics, Strongly Correlated Materials, Superconductivity

and cuprate superconductors [3], and the mechanisms behind it are therefore a topic of interest.

In many iron-based superconductors, nematicity occurs in close proximity to a transition into unidirectional spin density wave order, suggesting that it is a melted form of the magnetic order $[4,5]$. In contrast, the nematic transition of FeSe occurs, at $92 \mathrm{~K}$, without a subsequent magnetic transition. Whereas in other iron-based superconductors magnetic and lattice fluctuations are linked by a scaling relationship, they are not so linked in FeSe [6-8]. In spite of these differences, there are similarities between FeSe and other iron-based superconductors that suggest that their nematicities are related. For example, unidirectional magnetic order can be induced in FeSe $[9,10]$, and the nematic electronic structure as observed in angle-resolved photoemission qualitatively matches that of $\mathrm{BaFe}_{2} \mathrm{As}_{2}[11,12]$. $\mathrm{FeSe}$ is a valuable reference material not only because of the absence of magnetic order, but also because of the absence of intrinsic dopant disorder, and the availability of high-quality, vapor-transport-grown samples [13,14]. 
Measurements of the strain dependence of resistivity, i.e., the elastoresistivity, have shown that the nematicity of FeSe, like that of other iron-based superconductors, is an electronic instability. The key observation is that the resistive anisotropy $\left(\rho_{x x}-\rho_{y y}\right) /\left(\rho_{x x}+\rho_{y y}\right)$ varies with strain at a rate that diverges with cooling [15-19]. The resistive anisotropy is understood to be proportional to an underlying electronic anisotropy that can be quantified by a nematic order parameter $\psi$. On a clamped lattice, $\psi$ would transition to a nonzero value at a bare transition temperature $T_{s, 0}$, but the elastic compliance of the lattice raises the transition temperature to $T_{s}>T_{s, 0}$. For $T>T_{s}$, applied anisotropic strain $\varepsilon$ induces nonzero $\psi$ through electronlattice coupling, with a susceptibility $d \psi / d \varepsilon$ that diverges (with divergence temperature $T_{s, 0}$ ) as the sample is cooled. Therefore, because resistive anisotropy is proportional to $\psi$, its dependence on strain also steepens with cooling.

An assumption of a linear relationship between $\psi$ and resistive anisotropy has become deeply enough embedded that resistive anisotropy is often employed as a measure of $\psi$. Here, we explore elastoresistivity at large $|\psi|$, where the relationship becomes strongly nonlinear. FeSe is considered to be a Hund's metal, meaning that interorbital charge fluctuations are suppressed by Hund's coupling [20]. Strong evidence for the importance of orbital character is provided by the fact that the magnitude of the superconducting gap correlates closely with $y z$ orbital weight [21-23]. Many of the strain effects that we observe here are also consistent with a prominent role of the $y z$ orbital in electronic correlations, and we discuss how our data may constitute a test of theories of the nematicity of FeSe.

Two types of measurement are presented. (1) Resistive anisotropy is measured as a function of strain-induced nematicity at constant temperature $T \sim T_{s}$. (2) Strain tuning is employed to control the twinning as samples are cooled, allowing measurement of the intrinsic resistive anisotropy at temperatures below $T_{s}$. Although the $T$-dependent nematic resistive anisotropy has been reported previously for a few iron-based compounds [19,24-27], these previous measurements have relied upon assumptions that twin boundary resistance is negligible and/or that a sustained stress applied to detwin samples is weak enough not to substantially alter the electronic structure, even though the iron-based superconductors are extremely sensitive to uniaxial stress $[25,28]$. With strain tuning, samples can be held in a fully or partially detwinned state without sustained application of external anisotropic stress.

This paper is organized as follows. We first present our setup and methods, and then define the key parameters for discussion of elastoresistivity. We then present results for application of anisotropic strain with principal axes rotated by $45^{\circ}$ from the nematic axes, in other words, where it constitutes a transverse field to the nematicity [29]. Results are then presented for strain aligned with the nematic axes, where the response is much stronger. Our main results, the spontaneous (a)

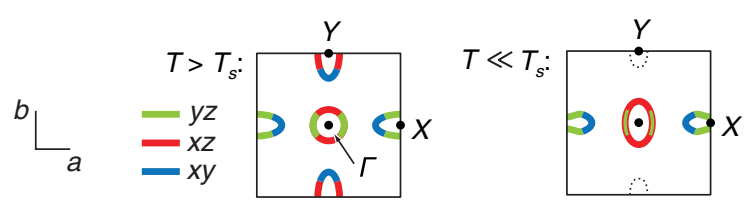

(b)
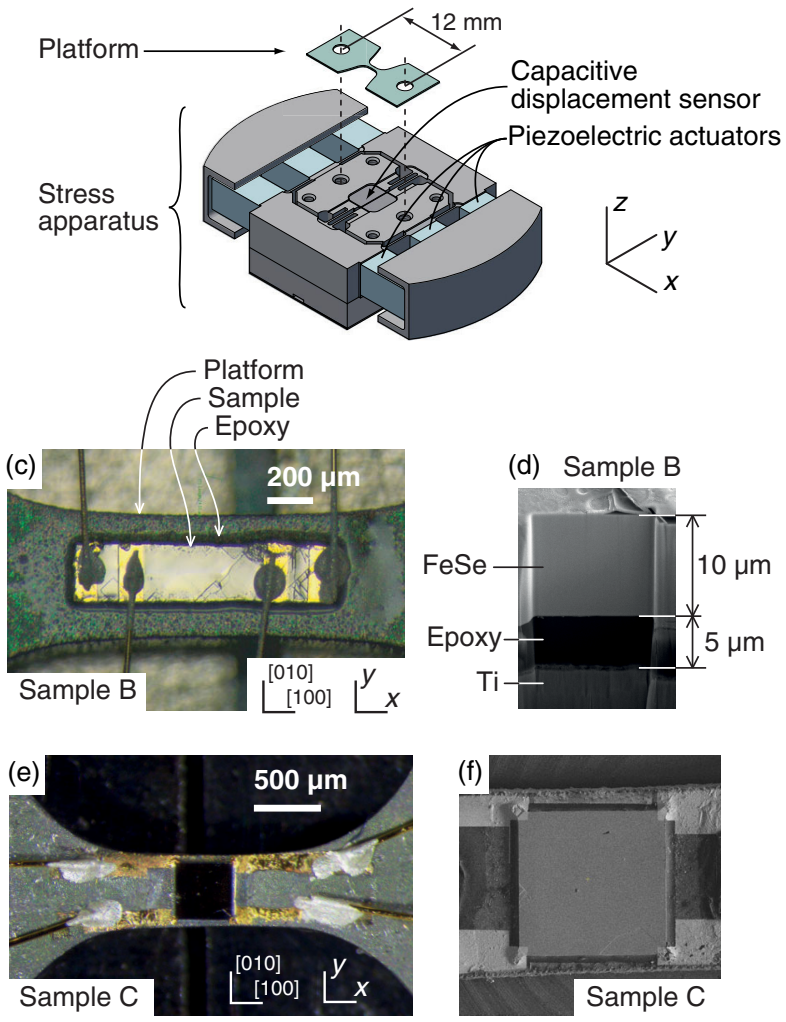

FIG. 1. (a) Schematic illustration of the electronic structure above and below the nematic transition temperature $T_{s}$. Fermi surfaces are colored by their dominant orbital content. For $T<T_{s}, k_{x}$ is oriented along the crystalline $a$ axis, where $a>b$. (b) Piezoelectric uniaxial stress apparatus with a platform. (c) Photograph of sample B, with contacts attached for measuring resistivity along the sample's length. The lattice directions are indicated. Sample A was prepared similarly, though with its crystal axes rotated by $45^{\circ}$. (d) Scanning electron (SEM) micrograph of a cut, made with a focused ion beam, through sample B and the epoxy layer beneath it. (e) Photograph and (f) SEM micrograph of sample $\mathrm{C}$, which was prepared in a Montgomery configuration.

nematic resistive anisotropy for $T<T_{s}$ and its comparison with that induced by strain at $T>T_{s}$, are shown in Fig. 8.

For orientation, the electronic structure of FeSe above and far below $T_{s}$ is illustrated schematically in Fig. 1(a). We work with the 1-Fe unit cell, in which the Fe-Fe bond directions, and the principal axes of the nematicity, are the $\langle 100\rangle$ directions. In the corresponding Brillouin zone, there is a hole pocket at the $\Gamma$ point, and two electron pockets, one at the $X$ and the other at the $Y$ point. In the nematic state, where the $a$ lattice parameter becomes larger than $b$, the pocket at $X$ distorts into a peanutlike shape elongated along $k_{x}$, signatures of the $Y$ pocket disappear from 
spectroscopic probes $[17,21,30-33]$, and the hole pocket becomes elongated along the $k_{y}$ direction.

\section{METHODS}

To apply large strains to FeSe, we affix samples to platforms with a layer of epoxy (Masterbond ${ }^{\circledR}$ EP29LPSP), and then apply stress to the platform; details of this method are presented in Ref. [34]. By preventing samples from buckling under compressive strain, the platform allows samples to be very thin. This is helpful for FeSe because it is a layered compound with a very low elastic limit for interlayer shear stress, which is minimized when samples are thin. The epoxy that wicked up the sides of the sample may also have served to hinder cleavage. A schematic of the setup is shown in Fig. 1(b), and images of mounted samples are shown in Figs. 1(c)-1(f).

Here, the platforms are titanium sheets. The central portion is cut into a narrow neck within which stress is concentrated, and samples are attached to this neck. The platforms are then mounted onto a piezoelectric-driven uniaxial stress apparatus. This apparatus incorporates a capacitive sensor of the applied displacement, and therefore of the longitudinal strain within the neck.

We report data from three samples. Sample A was cut for application of strain with $\langle 110\rangle$ principal axes, and samples B and C with $\langle 100\rangle$ principal axes. Samples A and B were prepared as shown in Fig. 1(c): bars with high lengthto-width ratio, with contacts for measurement of resistivity along the sample length. Sample C, shown in Figs. 1(e) and 1(f), was prepared in a Montgomery configuration for simultaneous measurement of longitudinal and transverse resistivities, as introduced for elastoresistivity measurements in Ref. [16]. The conversion from measured resistances to longitudinal and transverse resistivities in the Montgomery geometry is discussed in Appendix A 1. In Appendix A 2, we consider the mechanics of strain transmission from the platform to the sample, and show that the lengths and widths of the samples here are all long enough that to good precision both the longitudinal and transverse strains can be taken to be locked to those in the platform.

Electrical contacts, fabricated from sputtered gold with no adhesion layer, were deposited on the samples' upper surfaces. The resistivity ratio $\rho_{c} / \rho_{a b}$ of FeSe appears not to have been measured; however, that of $\mathrm{FeSe}_{0.4} \mathrm{Te}_{0.6}$ is $\approx 70$ at $15 \mathrm{~K}$ [35]. The length scale for current injected at the upper surface to spread out over the full sample thickness is $t\left(\rho_{c} / \rho_{a b}\right)^{1 / 2}$, where $t \sim 10 \mu \mathrm{m}$ is the sample thickness. This length scale is short enough that measurements here are not strongly affected by the $c$-axis resistivity. For sample $\mathrm{C}$, the contacts also run down the sides of the sample.

\section{STRAIN PARAMETERS}

The applied strain can be resolved into symmetric and antisymmetric components, and throughout this work it
TABLE I. Strain parameters. We take the 1-Fe unit cell, in which the $\langle 100\rangle$ directions are Fe-Fe bond directions. Sample A is aligned so that stress is applied along the [110] lattice direction; the strain along this axis, $\varepsilon_{110}$, is measured by the displacement sensor integrated into the stress cell. Samples B and C are aligned so that stress is applied along the [100] direction. $\nu=0.32$ is the Poisson ratio of the platform. The graphics illustrate the strain directions. We take the sign convention that $\varepsilon<0$ denotes compression.

\begin{tabular}{|c|c|}
\hline \multicolumn{2}{|r|}{ Sample A } \\
\hline (1) & $\begin{array}{l}\varepsilon_{A 1 g} \equiv \frac{1}{2}\left(\varepsilon_{110}+\varepsilon_{1 \overline{1} 0}\right)=\frac{1}{2}(1-\nu) \varepsilon_{110}=0.34 \varepsilon_{110} \\
\varepsilon_{B 2 g} \equiv \frac{1}{2}\left(\varepsilon_{110}-\varepsilon_{1 \overline{1} 0}\right)=\frac{1}{2}(1+\nu) \varepsilon_{110}=0.66 \varepsilon_{110}\end{array}$ \\
\hline \multicolumn{2}{|r|}{ Samples B and C } \\
\hline 1 & $\begin{array}{l}\varepsilon_{A 1 g} \equiv \frac{1}{2}\left(\varepsilon_{100}+\varepsilon_{010}\right)=\frac{1}{2}(1-\nu) \varepsilon_{110}=0.34 \varepsilon_{100} \\
\varepsilon_{B 1 g} \equiv \frac{1}{2}\left(\varepsilon_{100}-\varepsilon_{010}\right)=\frac{1}{2}(1+\nu) \varepsilon_{110}=0.66 \varepsilon_{010}\end{array}$ \\
\hline
\end{tabular}

will be important to resolve their separate effects. Here, we define quantities for discussion. For sample A, stress is applied along the [110] lattice direction; the displacement sensor in the stress cell measures the strain along this axis, $\varepsilon_{110}$. The transverse strain $\varepsilon_{1 \overline{1} 0}$ is given by $\varepsilon_{1 \overline{1} 0}=-\nu \varepsilon_{110}$, where $\nu=0.32$ is the Poisson ratio of the platform. The symmetric component of the strain field is $\varepsilon_{A 1 g} \equiv \frac{1}{2}\left(\varepsilon_{110}+\varepsilon_{1 \overline{1} 0}\right)$, which comes to $0.34 \varepsilon_{110}$, while the antisymmetric component is $\varepsilon_{B 2 g} \equiv \frac{1}{2}\left(\varepsilon_{110}-\varepsilon_{1 \overline{1} 0}\right)=$ $0.66 \varepsilon_{110}$. These parameters, along with equivalent parameters for samples B and C, are summarized in Table I. We also label resistivities by the measurement axis: $\rho_{100}$, for example, is the resistivity along the [100] direction. For sample $\mathrm{C}$, both $\rho_{100}$ and $\rho_{010}$ are measured, and so symmetric and antisymmetric resistivities can be defined: $\rho_{A 1 g} \equiv \frac{1}{2}\left(\rho_{100}+\rho_{010}\right)$ and $\rho_{B 1 g} \equiv \frac{1}{2}\left(\rho_{100}-\rho_{010}\right)$.

We note that specifying lattice distortions becomes more complicated when the lattice twins. We adopt here the convention that [110] for sample A and [100] for samples B and $\mathrm{C}$ always refer to the direction along the length of the platform. When the sample twins, we use $a$ and $b$ to refer to the directions along which the in-plane lattice constant lengthens and shrinks, respectively; in other words, the $a$ and $b$ axes are defined locally, and the [100] and [110] directions globally.

For all samples, the applied strain will also generate a $c$-axis strain in the sample, $\varepsilon_{001}=-2 c_{13} \varepsilon_{A 1 g} / c_{33}$. $c$-axis strain preserves the tetragonal symmetry of the $T>T_{s}$ lattice, and therefore is in the $A_{1 g}$ representation. When we discuss $A_{1 g}$ strain it should be understood that it includes this associated $c$-axis strain.

Because the aim of this work is to explore the nonlinear regime, we do not apply the elastoresistivity matrix formalism introduced in Ref. [36]. For comparison with previous results, we note that the quantity $\left(1 / \rho_{A 1 g}\right) d \rho_{B 1 g} / d \varepsilon_{B 1 g}$ at $\varepsilon_{B 1 g}=0$ is equal to $m_{11}-m_{12}$ in that formalism. Most 
previous elastoresistivity results have been reported using the 2-Fe unit cell, in which $m_{11}-m_{12}$ transforms to $2 m_{66}$.

\section{RESULTS: $\langle 110\rangle$ STRAIN}

Although strong transverse strain is predicted to enhance quantum fluctuations and suppress nematicity [29,37], the range of transverse strain explored here shifts $T_{s}$ by only a few kelvin. $T_{s}$ can be identified from an upturn in the resistivity, and, as shown in Fig. 2, decreases at a modest rate with compression. Within our strain range only a linear component of the strain dependence is resolved, with slope $d T_{s} / d \varepsilon_{110}=750 \mathrm{~K}$. This slope is due to the $A_{1 g}$ component of the applied strain: under the tetragonal symmetry of FeSe at $T>T_{s}$, reversal of the sign of $\varepsilon_{B 2 g}$ gives a symmetrically equivalent strain, so coupling to $\varepsilon_{B 2 g}$ can give only strain-even components in the strain dependence of $T_{s}$.

$\varepsilon_{A 1 g}=0.34 \varepsilon_{110}$, so $d T_{s} / d \varepsilon_{110}=750 \mathrm{~K}$ corresponds to $d T_{s} / d \varepsilon_{A 1 g}=2200 \mathrm{~K}$. In Ref. [38], $T_{s}$ is found to be suppressed by compressive hydrostatic stress with an initial slope of $39 \mathrm{~K} / \mathrm{GPa}$. Using the elastic moduli of Ref. [39], this converts to $d T_{s} / d \varepsilon_{A 1 g} \approx 6200 \mathrm{~K}$. (See Appendix A 3 for details.) The difference between this and our result allows, in principle, separation of the effect of $c$-axis strain $\varepsilon_{001}$ and that of "pure" in-plane biaxial strain $\varepsilon_{A 1 \text { g,pure }}$ that has no associated $c$-axis strain. Applying again the elastic moduli from Ref. [39], under in-plane uniaxial stress $\varepsilon_{001}=-0.3 \times \varepsilon_{A 1 \text { g,pure }}$, and under hydrostatic stress $\varepsilon_{001}=1.0 \times \varepsilon_{A 1 g, \text { pure }}, \quad$ so $\quad \Delta T_{s} \approx(3200 \mathrm{~K}) \times \varepsilon_{A 1 \text {,pure }}+$ $(1000 \mathrm{~K}) \times \varepsilon_{001}$.

\section{RESULTS: $\langle 100\rangle$ STRAIN}

\section{A. Stress-temperature versus strain-temperature phase diagram}

The effect of strain applied along the principal axes of the nematicity is much more dramatic. Before showing results, we discuss the differences between stress- and strain-temperature phase diagrams for a nematic transition. The distinction between stress and strain is equivalent to that between magnetic field $H$ and magnetic induction $B$. When a ferromagnet is cooled through its Curie temperature under nonzero $H$, the transition broadens into a crossover. Experimentally, controlled $H$ is applied by preparing samples to have a low demagnetization factor: thin bars parallel to the applied field. In the opposite limit, of a thin plate perpendicular to the applied field, it is $B$ that is held fixed, and if $B / \mu_{0}$ is less than the spontaneous magnetization $M$ of the sample, then in general magnetic domains will form such that the sample's average magnetization matches the applied $B$. Domain formation under nonzero applied $B$ requires reversal of local magnetization, so it is a first-order transition rather than a crossover.
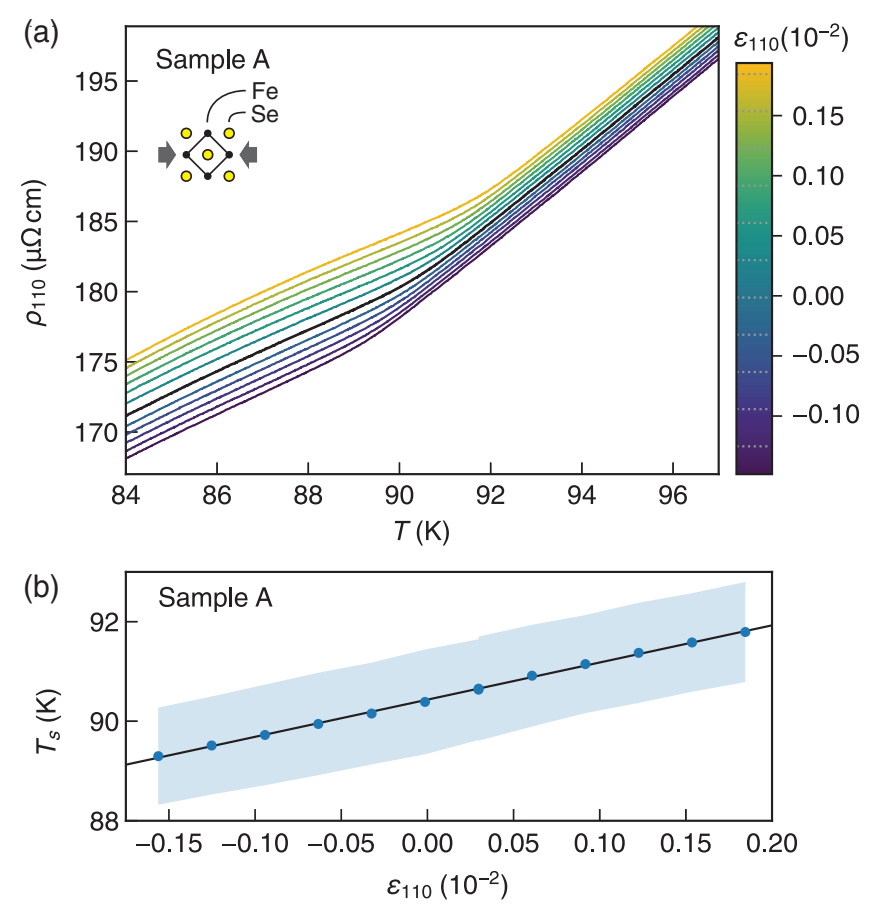

FIG. 2. The effect of transverse strain. (a) $\rho_{110}(T)$, the resistivity along the [110] direction, for sample $\mathrm{A}$ at various applied strains $\varepsilon_{110}$. The principal axes of the nematicity in FeSe are the $\langle 100\rangle$ axes, so this strain is a transverse field to the nematicity. Inset: schematic of the strain axis. (b) $T_{s}$ versus $\varepsilon_{110}$ for this sample. $T_{s}$ is identified as the maximum in $d^{2} \rho_{110} / d T^{2}$. The shaded region is a measure of the width of the transition: it is where $d^{2} \rho / d T^{2}$ exceeds half its maximum value. The line is a fit.

For nematic compounds, the difference between stress and strain is illustrated in Fig. 3. In the stress-temperature phase diagram, a first-order transition line corresponding to reversal of the nematicity runs along the zero-stress axis from $T=T_{s}$ to $T \rightarrow 0$. In the strain-temperature phase

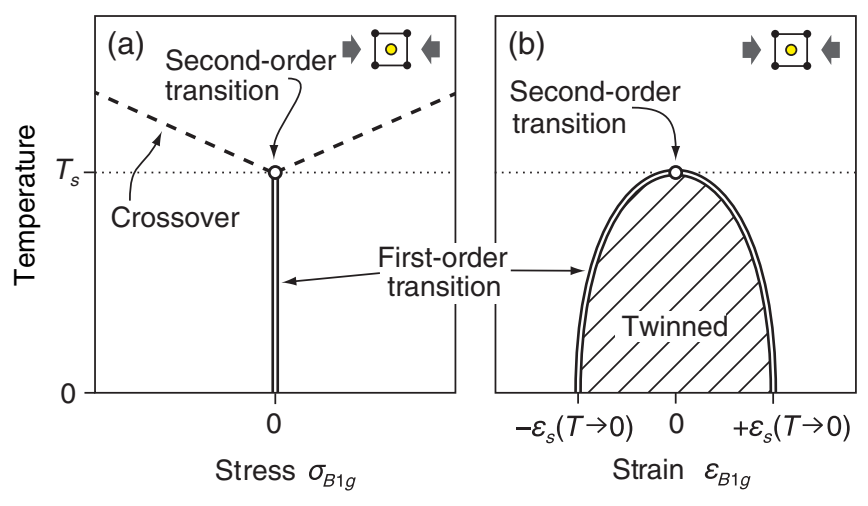

FIG. 3. Schematic phase diagrams. (a) Schematic stress-temperature phase diagram for the nematicity of FeSe, for stress applied with $B_{1 g}$ principal axes; the first-order transition is where the direction of the nematicity flips. (b) The corresponding straintemperature phase diagram. In the indicated region, the lattice is unstable and breaks up into twins where, locally, $\varepsilon_{B 1 g}= \pm \varepsilon_{s}(T)$. 
diagram, on the other hand, there are two lines of first-order transitions. The structural distortion in FeSe is to high precision a $B_{1 g}$ distortion, meaning that $b$ contracts by nearly the same amount as $a$ lengthens [40-42]. Therefore, the nematicity-induced structural distortion can be described as a spontaneous local strain $\varepsilon_{B 1 g \text {,local }}= \pm \varepsilon_{s}(T)$, where the quantity $\varepsilon_{s}$ is termed the structural strain. The average strain in the sample must match that of the platform, but when $\left|\varepsilon_{B 1 g}\right|<\varepsilon_{S}(T)$ twin formation is favored, and the applied strain sets the equilibrium twin volume ratio. Like formation of magnetic domains under nonzero $B$, formation of twinned domains under nonzero applied $\varepsilon_{B 1}$ is a first-order process, so the twinned region is bounded by first-order transitions.

In the stress-temperature phase diagram there will be resolvable crossover lines at $T>T_{s}$ : when the applied stress is small, there will be a small temperature range over which the nematicity-driven strain increases at a rapid but nondivergent rate. In this sense, stress acts as a classic conjugate field. We present some evidence below on whether equivalent crossover lines are discernable in the strain-temperature phase diagram.

\section{B. Sample B: $T \sim T_{s}$}

Measurements of resistivity confirm this qualitative form of strain-temperature phase diagram. To facilitate comparison with measurements of $\varepsilon_{s}$, we now plot data against the antisymmetric strain $\varepsilon_{B 1 g} . \rho_{100}\left(\varepsilon_{B 1 g}\right)$ of sample B for $T \sim T_{s}$ is shown in Fig. 4(a), and the derivative $d \rho_{100} / d \varepsilon_{B 1 g}$ in Fig. 4(b). The neutral strain point $\varepsilon_{B 1 g}=0$ is determined as the strain where the twin boundary density for $T<T_{s}$ is highest; these data are shown below. Above $T_{s}$, the strain dependence of $\rho_{100}$ is seen to have substantial nonlinearity even over a relatively small strain range $\left|\varepsilon_{B 1 g}\right|<0.1 \times 10^{-2}$. Its slope is largest near, though not precisely at, $\varepsilon_{B 1 g}=0$.

As $T$ is reduced below $T_{s}$, the onset of twinning changes the form of $\rho_{100}\left(\varepsilon_{B 1 g}\right)$ : a range of strain appears over which $d \rho_{100} / d \varepsilon_{B 1 g}$ becomes nearly constant. This change is easiest to see in Fig. 4(b), where we have marked the twinned region for the $86.8 \mathrm{~K}$ curve. The origin of this behavior is illustrated schematically in Fig. 4(c). When the sample twins, within each twin domain the resistivities along the local $a$ and $b$ axes are $\rho_{a}$ and $\rho_{b}$, and the equilibrium twin volume ratio is a linear function of applied strain. Therefore, the observed bulk resistivity is an interpolation between $\rho_{b}$ at $\varepsilon_{B 1 g}=-\varepsilon_{s}$ and $\rho_{a}$ at $\varepsilon_{B 1 g}=+\varepsilon_{s}$, that to high precision is linear under two conditions that are both satisfied here. (1) $\mid\left(\rho_{a}-\rho_{b}\right) /$ $\left(\rho_{a}+\rho_{b}\right) \mid$ is much less than 1 , so that redistribution of current into lower-resistivity domains does not substantially alter the observed bulk resistivity. (2) The domain wall resistance is negligible, which we show later to be the case for $T$ near $T_{s}$.

Even though the transitions into the twinned region must, when $\varepsilon_{B 1 g} \neq 0$, be first order, no hysteresis is resolved,
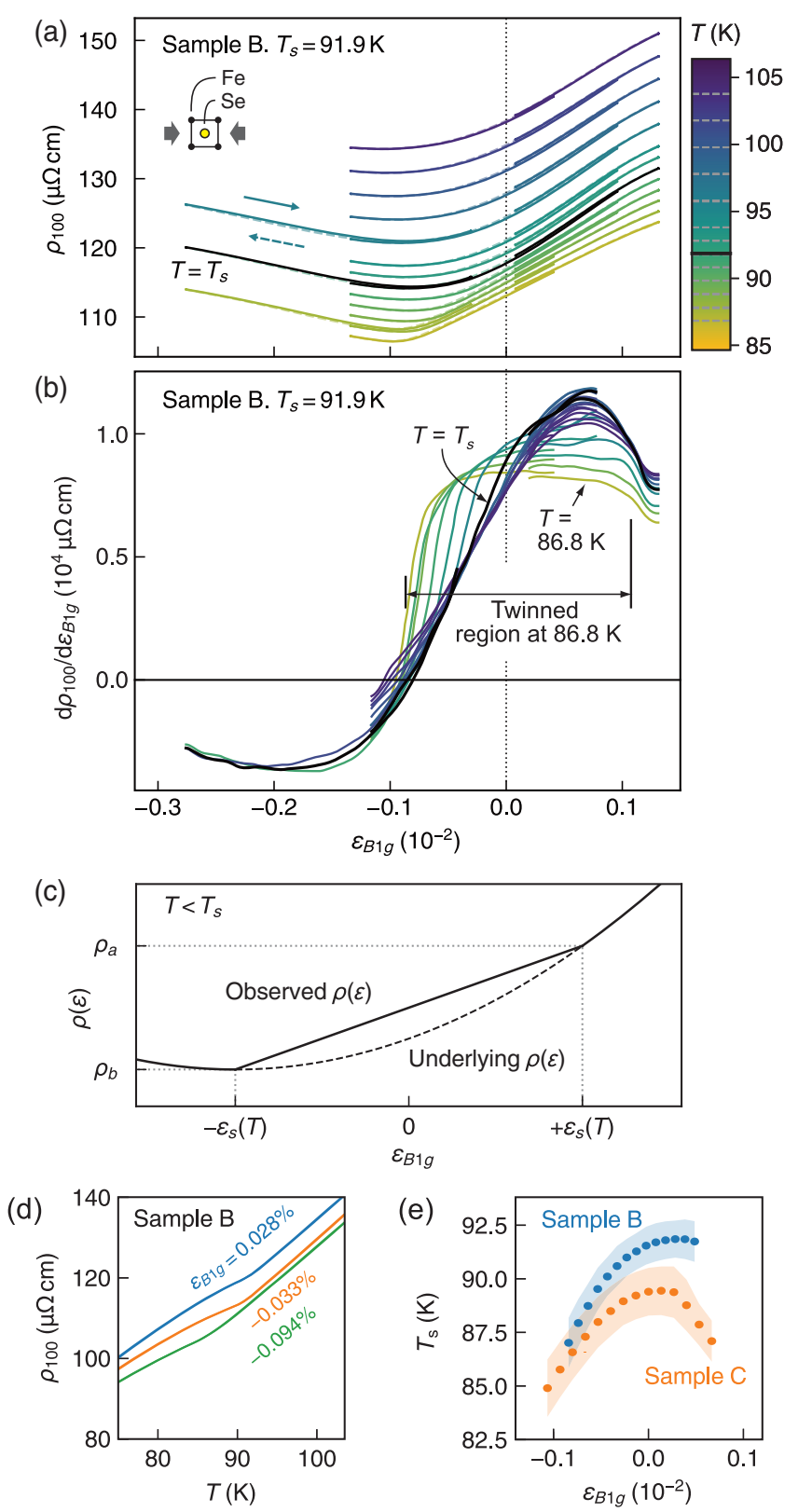

FIG. 4. Elastoresistivity near $T_{s}$. (a) $\rho_{100}\left(\varepsilon_{B 1 g}\right)$, where $\rho_{100}$ is the resistivity along the [100] direction and $\varepsilon_{B 1 g} \equiv\left(\varepsilon_{100}-\varepsilon_{010}\right) / 2$, of sample B at various temperatures near $T_{s}$. (b) $d \rho_{100} / d \varepsilon_{B 1 g}$ for the curves from (a). For $T<T_{s}, d \rho_{100} / d \varepsilon_{B 1 g}$ becomes nearly constant over the range where the sample twins. This range is indicated for the $86.8 \mathrm{~K}$ curve. (c) Schematic of $\rho_{100}\left(\varepsilon_{B 1 g}\right)$ for $T<T_{s}$; the underlying curve is not accessible for $-\varepsilon_{s}<\varepsilon_{B 1 g}<+\varepsilon_{s}$ due to the onset of twinning, and the observed resistivity instead interpolates over this range. (d) Temperature ramps at three values of $\varepsilon_{B 1 g}$. (e) $T_{s}$ versus strain for low strains. The shaded regions indicate the transition width, defined by $d^{2} \rho_{100} / d T^{2}$ crossing half its maximum value.

indicating that the energy barrier for twin formation is low. Separately, close inspection of Fig. 4(b) reveals that twinning does not initially onset right at $\varepsilon_{B 1 g}=0$, but 
slightly on the tensile side. This asymmetry is due to the $A_{1 g}$ component of the applied strain: as shown with sample A in Fig. 2, tensile $A_{1 g}$ strain increases $T_{s}$.

$\rho$ versus temperature at a few nonzero $\varepsilon_{B 1 g}$ are shown in Fig. 4(d), and Fig. 4(e) shows $T_{s}$ derived from such temperature sweeps as a function of strain. For both samples B and C, $T_{s}$ follows a downward quadratic form, consistent with the schematic strain-temperature phase diagram illustrated in Fig. 3(b).

\section{Sample B: $T<T_{s}$}

Figure 5(a) shows $\rho_{100}$ of sample B over a much wider temperature and strain range. Here, the contribution of twin boundaries to the total sample resistance becomes apparent. Two datasets are shown: strain ramps in which $T$ was incremented at $\varepsilon_{B 1 g}<-\varepsilon_{S}(T)$ and temperature ramps in which strain was incremented at $T>T_{s}$. The maximum compression reached was $\varepsilon_{B 1 g}=-0.28 \times 10^{-2}$, which exceeds the spontaneous $T \rightarrow 0$ structural distortion of FeSe and fully detwins the sample at all temperatures. It corresponds to a longitudinal strain of $\varepsilon_{100}=$ $-0.42 \times 10^{-2}$, and was large enough to exceed the elastic limit of the platform. Plastic deformation of the platform introduced an anomalous offset between $\varepsilon_{B 1 g}$ and $\varepsilon_{A 1 g}$ at large strains. Data in Appendix A 4, where the plastic deformation is described in more detail, show that the resistivity of $\mathrm{FeSe}$ depends much more sensitively on $\varepsilon_{B 1 g}$ than $\varepsilon_{A 1 g}$, and so we continue to plot data against $\varepsilon_{B 1 g}$. Crucially, the sample residual resistivity did not change, showing that its own deformation remained elastic even as the platform deformed plastically.

For $T$ above $\approx 60 \mathrm{~K}$, the structural strain $\varepsilon_{s}(T)$ can be identified by a sharp change in slope $d \rho_{100} / d \varepsilon_{B 1 g}$, as seen also in Figs. 4(a) and 4(b). To obtain $\varepsilon_{s}$ at all temperatures, we scale $\varepsilon_{s}(T)$ from the x-ray diffraction data of Ref. [40] in temperature to match $T_{s}$ of this sample, and in strain to match the locations of the cusps. This procedure gives $\varepsilon_{s}(T \rightarrow 0)=0.22 \times 10^{-2}$. For comparison, $\varepsilon_{s}(T \rightarrow 0)=$ $0.27 \times 10^{-2}$ and $0.23 \times 10^{-2}$ were obtained, respectively, in Refs. [40,43] by $x$-ray diffraction, $0.24 \times 10^{-2}$ and $0.25 \times 10^{-2}$ in Refs. [44,45] by neutron scattering, and $0.22 \times 10^{-2}$ in Ref. [13] by dilatometry measurements.

Figure 5(b) shows $\rho_{100}(T)$ at fixed strain $\varepsilon_{B 1 g}=-0.25 \times$ $10^{-2}$, where the sample is detwinned at all temperatures. $\rho_{100}$ evolves smoothly from $T_{c}$ to above $T_{s}$, with no feature apparent that could be identified as a nematic crossover. In other words, it does not appear to be useful to consider strain as a conjugate field to nematicity in FeSe, because even under a strain that is only barely large enough to detwin the sample, any nematic crossover appears to be so broad as to be indistinguishable from the background.

We now discuss twin boundaries. For $\left|\varepsilon_{B 1 g}\right|<\varepsilon_{s}(T)$, $\rho_{100}$ from the temperature ramps systematically exceeds that from the strain ramps. Figure 5(c) shows a close-up of
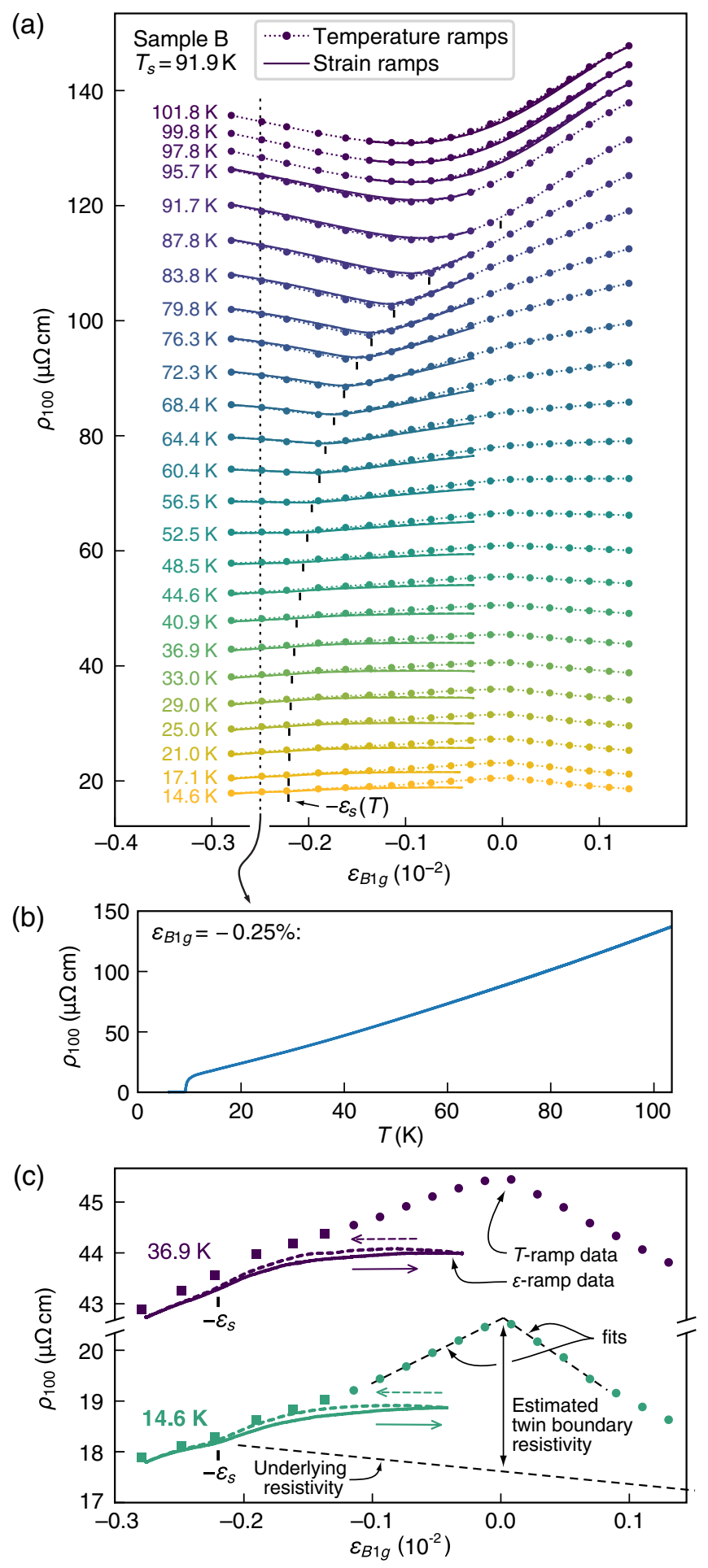

FIG. 5. (a) $\rho_{100}$ of sample B over a wide temperature and strain range. Points are data from temperature ramps at constant $\varepsilon_{B 1 g}$. The positions of points at $\epsilon_{B 1 g}<-0.11 \cdot 10^{-2}$ have been adjusted along the $\epsilon_{B 1 g}$ axis to correct for plastic deformation of the platform; see Appendix A 4 for details. The lines are strain ramps at constant temperature. $\varepsilon_{s}(T)$, taken as the data of Ref. [40] scaled in $T$ and $\varepsilon$ to match the data here, is indicated at each temperature. (b) $\rho_{100}(T)$ for $\varepsilon_{B 1 g}=-0.25 \times 10^{-2}$, where the sample is fully detwinned at all $T$. (c) Close-up of the data in (a) at 14.6 and $36.9 \mathrm{~K}$. The squares mark points whose position along the $\varepsilon_{B 1 g}$ axis was corrected. 
data at 36.9 and $14.6 \mathrm{~K}$ : the $T$-ramp data have a peaked form that the strain-ramp data do not. The magnitude of this peak is very similar at the two temperatures, even though the intrinsic resistivity at $36.9 \mathrm{~K}$ is more than double that at $14.6 \mathrm{~K}$, which shows that its origin is extrinsic. It is due to twin boundaries. The elastic mismatch between the sample, which distorts orthorhombically, and the platform, which does not, will be strongest at $\varepsilon_{B 1 g}=0$, leading to a peak in the equilibrium twin boundary density. This peak is resolvable for temperatures up to $\sim 70 \mathrm{~K}$, at a temperature-independent strain, which we therefore identify as the neutral strain point $\varepsilon_{B 1 g}=0$. Evidence for twinning is also directly visible in the strainramp data in Fig. 5(c); there is hysteresis for $\left|\varepsilon_{B 1 g}\right|<\varepsilon_{s}$ that closes when $\left|\varepsilon_{B 1 g}\right|>\varepsilon_{s}$. In Appendix A 5 we show that ramping the strain back and forth can partially anneal twin boundaries out of the sample.

A method to estimate the twin boundary contribution to the measured resistivity is illustrated in Fig. 5(c). For a $B_{1 g}$ lattice distortion, the twin boundary density is expected to be symmetric about $\varepsilon_{B 1 g}=0$. Furthermore, because twin boundaries are oriented along $\langle 110\rangle$ directions [19], no average change in twin boundary orientation is expected for strain with $\langle 100\rangle$ principal axes. We therefore fit lines to the temperature-ramp data on either side of the cusp and average their slopes to obtain an underlying slope, meaning the slope $d \rho_{100} / d \varepsilon_{B 1 g}$ that would be observed if the twin boundary resistance were zero. The line labeled "underlying resistivity" in Fig. 5(c) is a line of this slope placed to intersect the data at $\varepsilon_{B 1 g}=-\varepsilon_{S}$, where the sample is detwinned. In this way, we find that at 14.6 $\mathrm{K}$ the twin boundary contribution to the sample resistance is as high as $15 \%$, for this sample geometry. Twin boundary density may be lower for thicker and/or freestanding samples.

\section{Sample C}

In sample $\mathrm{C}$ both the longitudinal and transverse resistivities, $\rho_{100}$ and $\rho_{010}$, were measured. Results from strain ramps are shown in Fig. 6, and from $T$ ramps in Appendix A 6. The neutral strain point $\varepsilon_{B 1 g}=0$ was again taken as the strain where twin boundary density in the $T$-ramp data was highest. Around $\varepsilon_{B 1 g}=0$ and at temperatures near $T_{s}, \rho_{100}$ and $\rho_{010}$ vary strongly and oppositely with $\varepsilon_{B 1 g}$, confirming previous reports that the low-strain elastoresistivity of FeSe is dominantly in the $B_{1 g}$ channel $[18,19]$. Below $T_{s}$, the twinning transition at $\varepsilon_{B 1 g}=-\varepsilon_{s}(T)$ is broader than for sample B. Although this could indicate lower sample quality, we also note that strain inhomogeneity will generally be worse in a square sample geometry than in the linear geometry of sample B. To estimate $\varepsilon_{s}(T)$ for sample C, we scale $\varepsilon_{s}(T)$ reported in Ref. [40] in temperature to match the observed $T_{s}$ of sample $\mathrm{C}$, but we do not scale it in strain.
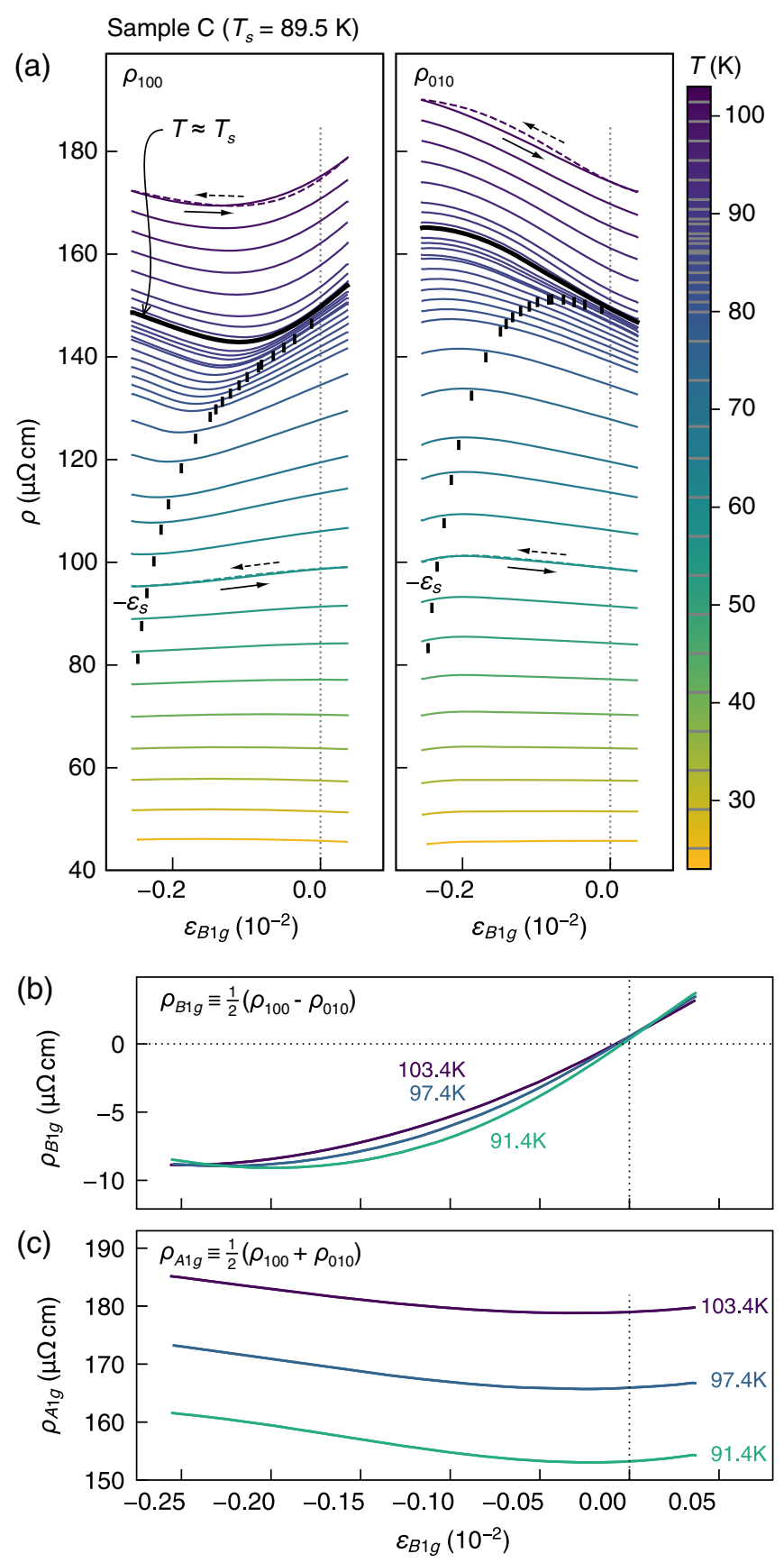

FIG. 6. Data from sample $\mathrm{C}$, the Montgomery configuration sample. (a) $\rho_{100}$ (left) and $\rho_{010}$ (right), from strain ramps at various fixed temperatures. The hysteresis is shown for two temperatures. The vertical ticks mark $-\varepsilon_{s}(T)$, taken from Ref. [40] and scaled in temperature to match the $T_{s}$ observed here. (b) $\rho_{B 1 g} \equiv\left(\rho_{100}-\rho_{010}\right) / 2$, derived from the data in (a), at temperatures above $T_{s}$. Note that by symmetry $\rho_{B 1 g}\left(T>T_{s}\right)=0$ at $\varepsilon_{B 1 g}=0$, but measurement error gives a small deviation from this. (c) $\rho_{A 1 g} \equiv\left(\rho_{100}+\rho_{010}\right) / 2$.

The antisymmetric resistivity $\rho_{B 1 g}$ for temperatures near $T_{s}$ is plotted in Fig. 6(b). Here it can be seen that although $\left|\rho_{B 1 g}\right|$ initially grows rapidly with strain-induced nematicity, it eventually reaches a maximum; just above $T_{s}$, this 
occurs at $\varepsilon_{B 1 g} \approx-0.19 \times 10^{-2}$. The symmetric resistivity $\rho_{A 1 g}$ is plotted in Fig. 6(c). For $T \gtrsim T_{s}, \rho_{A 1 g}$ is a minimum near $\varepsilon_{B 1 g}=0$, and as $T$ is reduced toward $T_{s}$ this minimum becomes sharper.

There are indications that other iron-based superconductors will have similar behavior. In $\mathrm{Ba}\left(\mathrm{Fe}_{0.975} \mathrm{Co}_{0.025}\right){ }_{2} \mathrm{As}_{2}$ (for which the nematicity also aligns with the $\langle 100\rangle$ directions), $\rho_{100}$ and $\rho_{010}$ both have upward curvature against $\varepsilon_{B 1 g}$, that grows sharper as $T$ is reduced to $T \approx T_{s}$ [46], suggesting that in this material too $\rho_{A 1 g}$ is a minimum for $\varepsilon_{B 1 g} \approx 0$. For $\mathrm{BaFe}_{2} \mathrm{As}_{2}, \rho_{100}$ near $T_{s}$ has been observed to have an $\mathrm{S}$-shaped dependence on $\varepsilon_{100}$, with the steepest slope appearing near $\varepsilon_{100}=0$ [47], matching the qualitative form (though with opposite sign) of $\rho_{100}\left(\varepsilon_{B 1 g}\right)$ observed here. Similar behavior is seen in $\mathrm{Sr}_{1-x} \mathrm{Ba}_{x} \mathrm{Fe}_{1.97} \mathrm{Ni}_{0.03} \mathrm{As}_{2}$ [48].

\section{EFFECT OF BIAXIAL STRAIN}

Data from sample $\mathrm{C}$ allow effects of the $A_{1 g}$ and $B_{1 g}$ strain components to be separated. The $A_{1 g}$ elastoresistivity $d \rho_{A 1 g} / d \varepsilon_{A 1 g}$ can be obtained by noting that within the twinned region $B_{1 g}$ strain does not couple locally to the sample, because the local $B_{1 g}$ strain is fixed at $\pm \varepsilon_{s}(T)$, but $A_{1 g}$ strain does couple locally. We take $\rho_{A 1 g}$ within the twinned region as $\rho_{A 1 g}=\left(\rho_{a}+\rho_{b}\right) / 2$, and now determine $d \rho_{A 1 g} / d \varepsilon_{A 1 g}$ at $\varepsilon_{B 1 g}=0$.

Under the approximation of linear interpolation between $\rho_{a}$ and $\rho_{b}$ and neglecting twin boundary resistance, $\rho_{100}$ and $\rho_{010}$ in the twinned region are given by

$$
\begin{aligned}
& \rho_{100}=f \rho_{a}+(1-f) \rho_{b}, \\
& \rho_{010}=f \rho_{b}+(1-f) \rho_{a},
\end{aligned}
$$

where $f=\left(\varepsilon_{s}+\varepsilon_{B 1 g}\right) / 2 \varepsilon_{s}$ is the volume fraction of the sample with the nematic $a$ axis oriented along the long axis of the platform. Differentiating with respect to $\varepsilon_{B 1 g}$ gives:

$$
\begin{aligned}
& \frac{d \rho_{100}}{d \varepsilon_{B 1 g}}=\frac{\rho_{a}-\rho_{b}}{2 \varepsilon_{s}}+f \frac{d \rho_{a}}{d \varepsilon_{B 1 g}}+(1-f) \frac{d \rho_{b}}{d \varepsilon_{B 1 g}}, \\
& \frac{d \rho_{010}}{d \varepsilon_{B 1 g}}=\frac{\rho_{b}-\rho_{a}}{2 \varepsilon_{s}}+f \frac{d \rho_{b}}{d \varepsilon_{B 1 g}}+(1-f) \frac{d \rho_{a}}{d \varepsilon_{B 1 g}} .
\end{aligned}
$$

Under the experimental conditions here, $d / d \varepsilon_{B 1 g}=$ $\left(d \varepsilon_{A 1 g} / d \varepsilon_{B 1 g}\right) d / d \varepsilon_{A 1 g}=[(1-\nu) /(1+\nu)] d / d \varepsilon_{A 1 g}$. Summing Eqs. (3) and (4) yields the $A_{1 g}$ elastoresistivity:

$$
\frac{d \rho_{A 1 g}}{d \varepsilon_{A 1 g}}=\frac{1+\nu}{2(1-\nu)}\left(\frac{d \rho_{100}}{d \varepsilon_{B 1 g}}+\frac{d \rho_{010}}{d \varepsilon_{B 1 g}}\right)
$$

To obtain underlying slopes $d \rho_{100} / d \varepsilon_{B 1 g}$ and $d \rho_{010} / d \varepsilon_{B 1 g}$, that is, that exclude the effect of twin boundaries, we
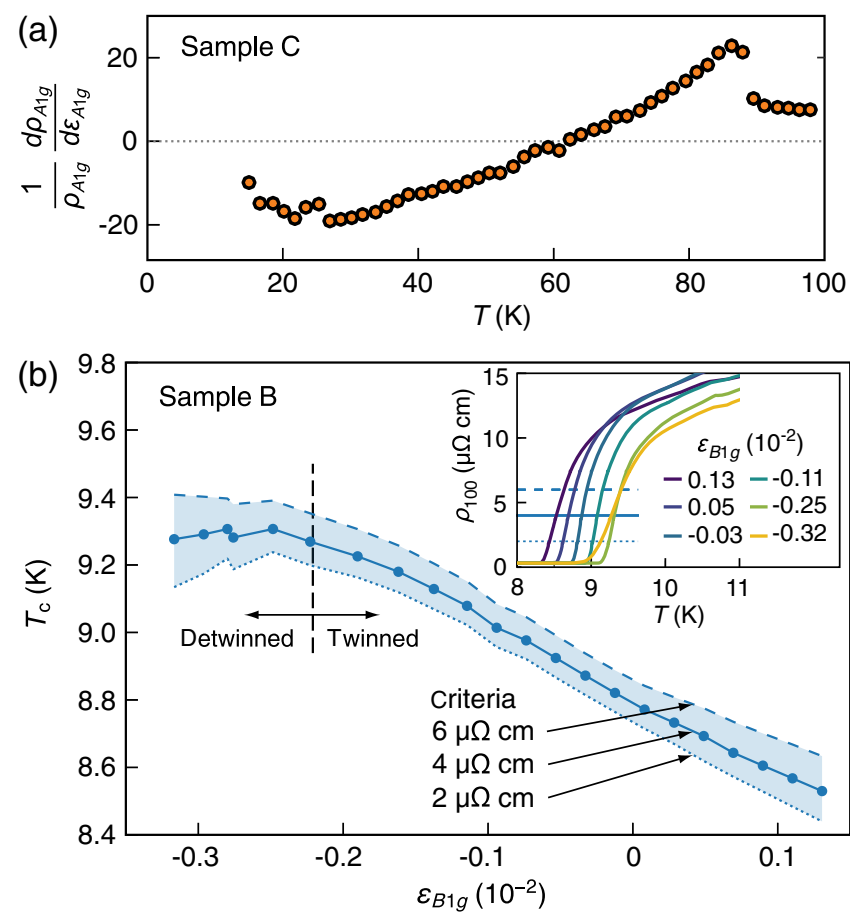

FIG. 7. Effect of biaxial strain. (a) $A_{1 g}$ elastoresistivity $\left(1 / \rho_{A 1 g}\right) d \rho_{A 1 g} / d \varepsilon_{A 1 g}$ versus $T$ of sample $\mathrm{C}$, determined as explained in the text. (b) $T_{c}$ versus strain, determined as the temperature where the resistivity crosses specific values, as shown in the inset. Note that within the twinned region, $\varepsilon_{B 1 g}$ does not couple locally to the sample, and instead the effect on $T_{c}$ is through the applied $A_{1 g}$ component of the strain. When the platform deformation is elastic, this is $\varepsilon_{A 1 g}=0.52 \varepsilon_{B 1 \mathrm{~g}}$. The observed slope therefore corresponds to $d T_{c} / d \varepsilon_{A 1 g}=-450 \mathrm{~K}$.

average the observed slopes on either side of $\varepsilon_{B 1 g}=0$, as shown in Fig. 5(c).

The $A_{1 g}$ elastoresistivity is shown in Fig. 7(a). It is normalized by $\rho_{A 1 g}$ at $\varepsilon_{B 1 g}=0$ with an estimate of the twin boundary resistance subtracted (see Appendix A 6 for details). For temperatures below $\approx 60 \mathrm{~K}, d \rho_{A 1 g} / d \varepsilon_{A 1 g}<0$, meaning that biaxial compression increases the average in-plane resistivity of FeSe. A similar temperature dependence is seen in the elastoresistivity of sample A; see Appendix A 7.

We show in Fig. 7(b), with data from sample B, that biaxial compression also increases $T_{c}$. (Again, when the sample is twinned only the $A_{1 g}$ component of the strain couples locally.) Both the increase in $T_{c}$ and $\rho_{A 1 g}$ are opposite to the generic expectation that compression should increase bandwidths. A similar correlation between resistivity and $T_{c}$ is also seen in strained $\mathrm{Sr}_{2} \mathrm{RuO}_{4}$ [49].

At large $\left|\varepsilon_{B 1 g}\right|$, the plastic deformation of the platform causes a gradual relaxation of the applied $A_{1 g}$ strain, and so for $\varepsilon_{B 1 g} \lesssim-0.15 \times 10^{-2}$, the $T_{c}$ curve bends downward subtly. For $\varepsilon_{B 1 g}<-\varepsilon_{s}$, the sample detwins, and the $B_{1 g}$ component of the applied strain couples locally to the 
sample. $T_{c}$ turns downward more sharply. Depending on the resistivity level selected as the criterion for $T_{c}$, it may even decrease. This behavior suggests that increasing the lattice orthorhombicity is detrimental to superconductivity.

\section{NEMATIC RESISTIVE ANISOTROPY}

We now report the central result of this paper, the nematic resistive anisotropy-both the spontaneous anisotropy below $T_{s}$ and that induced by strain at $T \sim T_{s}$. We obtain $\rho_{a}-\rho_{b}$ at $T<T_{s}$ by analyzing temperature-ramp data at small strains. At $\varepsilon_{B 1 g}=0, f$ in Eqs. (1) and (2) is 0.5 , yielding

$$
\rho_{a}-\rho_{b}=\varepsilon_{s}\left(\frac{d \rho_{100}}{d \varepsilon_{B 1 g}}-\frac{d \rho_{010}}{d \varepsilon_{B 1 g}}\right)
$$

The underlying slopes $d \rho_{100} / d \varepsilon_{B 1 g}$ and $d \rho_{010} / d \varepsilon_{B 1 g}$ are obtained, as before, by averaging the observed slopes from $\varepsilon_{B 1 g}>0$ and $<0$.

In Fig. 8(a) we show the nematic resistive anisotropy at $T<T_{s}$, normalized by $\rho_{A 1 g}$ (with, again, an estimate for the twin boundary resistivity subtracted; see Appendix A 6). Separate derivations from strain-ramp data from sample $\mathrm{C}$, and from data from sample $\mathrm{B}$, are shown in Appendix A 8; the agreement is excellent, which confirms that the twin boundary resistance has been properly canceled. The nematic resistive anisotropy peaks at $\approx 7 \%$, at $T \approx 80 \mathrm{~K}$, but then decreases as $T$ is reduced further, eventually changing sign at $\approx 40 \mathrm{~K}$. The low-temperature resistive anisotropy, where the nematicity is fully developed, is about $-1.5 \%$. This is surprisingly small: in angle-resolved photoemission spectroscopy data, the length-to-width ratios of the Fermi surfaces at $X$ and $\Gamma$ is 2-3 [22]. Any anisotropy in conduction from these Fermi surfaces individually appears to cancel almost perfectly. In contrast, resistive anisotropy in materials with magnetic order is much larger, for example, on the order of $100 \%$ in underdoped $\mathrm{Ba}(\mathrm{Fe}, \mathrm{Co})_{2} \mathrm{As}_{2}$ [24].

In Ref. [19], $\rho_{a}$ and $\rho_{b}$ were obtained by comparing the resistivities of stress-detwinned and unstressed samples, taking the former to be $\rho_{a}$ and the latter $\left(\rho_{a}+\rho_{b}\right) / 2$. $\left(\rho_{a}-\rho_{b}\right) /\left(\rho_{a}+\rho_{b}\right)$ was found to be $\approx 3 \%$, with weak temperature dependence, in qualitative disagreement with the results here. However, this analysis method treats the twin boundary resistance as negligible, which we have shown not to be a good approximation at lower temperatures.

This is, however, a valid approach near $T_{s}$, where twin boundary resistance is low compared with the total sample resistance. We show in the inset of Fig. 8(a) $\rho_{a}$ and $\rho_{b}$ of sample $\mathrm{C}$, derived by taking the $T$-ramp resistivity at $\varepsilon_{B 1 g}=0$ as $\left(\rho_{a}+\rho_{b}\right) / 2$ and then applying the anisotropy plotted in the main panel to obtain $\rho_{a}$ and $\rho_{b}$. Upon cooling into the nematic phase, $\rho_{b}$ is seen to decrease and $\rho_{a}$ to increase.
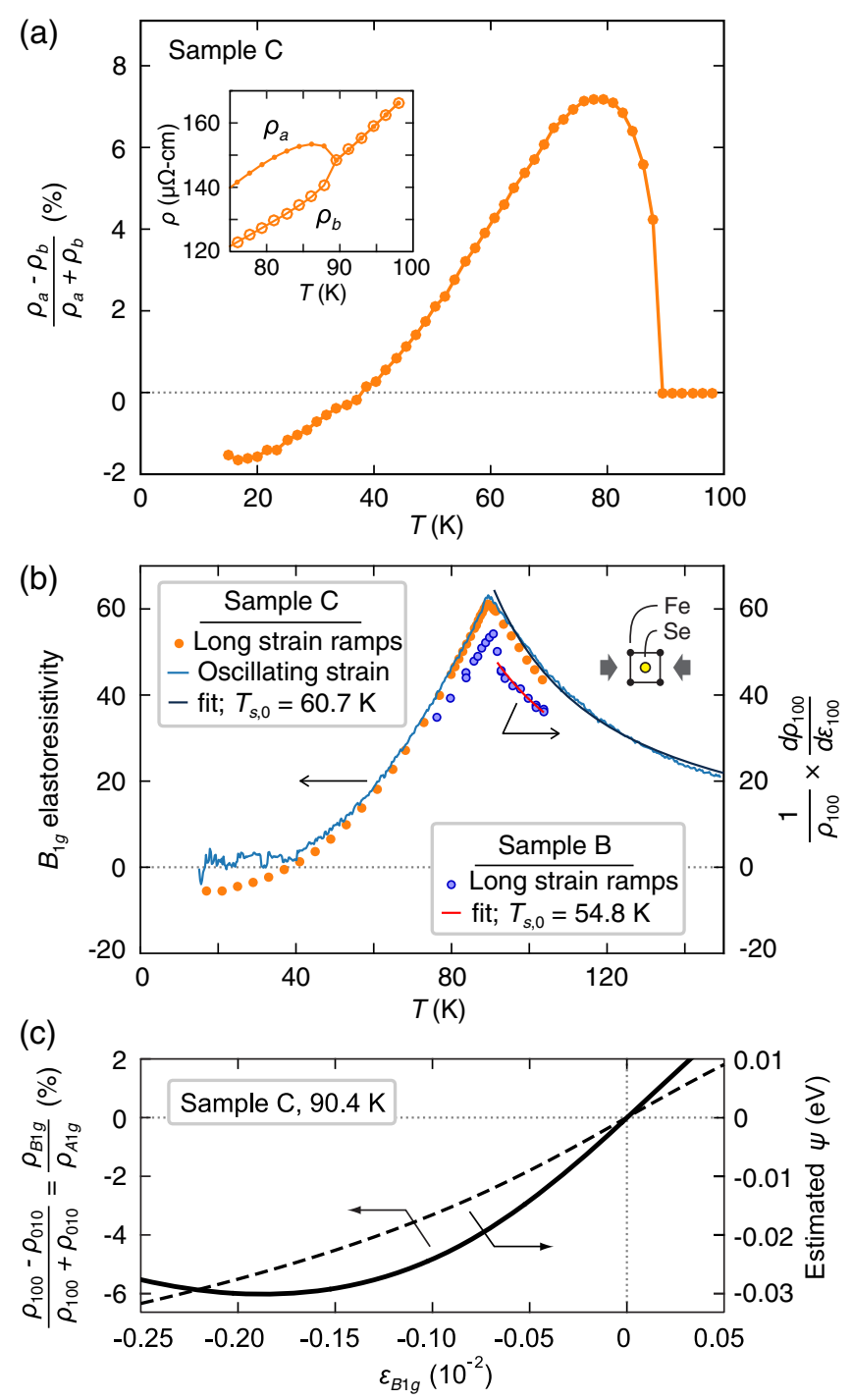

FIG. 8. Nematic resistive anisotropy. (a) The spontaneous resistive anisotropy below $T_{s}$, obtained from $T$-ramp data as described in the text. The inset shows $\rho_{a}$ and $\rho_{b}$ near $T_{s}$. (b) $B_{1 g}$ elastoresistivity, $\left(1 / \rho_{A 1 g}\right) d \rho_{B 1 g} / d \varepsilon_{B 1 g}$, obtained from both long strain ramps and from a small oscillating strain. For sample B, $\rho_{010}$ was not measured, so the [100] elastoresistivity $\left(1 / \rho_{100}\right) \times$ $d \rho_{100} / d \varepsilon_{100}$ is plotted instead. Fits are to a Curie-Weiss form; see the text. (c) Resistivity anisotropy of sample $\mathrm{C}$ against strain at $T \approx T_{s}$. The curve has been shifted vertically to set $\rho_{B 1 g}=0$ at $\varepsilon_{B 1 g}=0$, canceling a small geometrical error in the measurement. Also shown is an estimate of the strain-induced nematicity $\psi$, taken as the $x z-y z$ energy splitting at the $X$ point, obtained from evaluation of Ginzburg-Landau parameters.

In Fig. 8(b) we compare the $B_{1 g}$ elastoresistivity derived from the long strain ramps shown in Fig. 6(a) to that from a classic elastoresistivity measurement, also performed on sample $\mathrm{C}$, in which the strain was oscillated by a small amplitude (here, a peak-to-peak amplitude of $3.4 \times 10^{-5}$ at $0.0167 \mathrm{~Hz}$ ) and the resulting oscillation amplitude of the resistivity was measured. For the long strain ramps, the nematic resistive anisotropy at $T<T_{s}$ was determined 
by methods similar to those described above (see Appendix A 8 for details), and the $B_{1 g}$ elastoresistivity is taken as $\left(1 / \varepsilon_{s}\right) \times\left(\rho_{a}-\rho_{b}\right) /\left(\rho_{a}+\rho_{b}\right)$. For $T>T_{s}$ the $B_{1 g}$ elastoresistivity is taken as $\left(1 / \rho_{A 1 g}\right) \times d \rho_{B 1 g} / d \varepsilon_{B 1 g}$; these two definitions are equivalent at $T=T_{s}$. The smallamplitude elastoresistivity was taken as $\left(1 / \rho_{A 1 g}\right) \times$ $d \rho_{B 1 g} / d \varepsilon_{B 1 g}$ at all temperatures. Perhaps surprisingly, the small-amplitude elastoresistivity tracks the long-strainramp data to well below $T_{s}$, which shows that even with a very small strain oscillation amplitude, twin boundaries shift with the applied strain.

The $B_{1 g}$ elastoresistivity of sample $\mathrm{C}$ peaks at 62 . Previously reported values, from measurements in which samples were affixed directly to piezoelectric actuators, are 61 [19], 38 [18], and 300 [17]. We fit the small-amplitude data at $T>T_{s}$ to a Curie-Weiss form,

$$
\frac{1}{\rho_{A 1 g}} \frac{d \rho_{B 1 g}}{d \varepsilon_{B 1 g}}=\frac{a}{T-T_{s, 0}},
$$

which yields $T_{s, 0}=60.7 \mathrm{~K}$. A similar fit to data from sample B [where, because $\rho_{010}$ was not measured, we analyze the quantity $\left(1 / \rho_{100}\right) d \rho_{100} / d \varepsilon_{100}$ ] yields $T_{s, 0}=$ $54.8 \mathrm{~K}$. (We note that we do not include a high-temperature offset term in these fits, because doing so returns negative values, implying that in the $T \rightarrow \infty$ limit compression would cause resistivity to increase, which is not expected.)

In Fig. 8(c) we show the normalized resistive anisotropy of sample $\mathrm{C}$ as a function of strain at $T \approx T_{s}$. This peaks at $\approx 6 \%$, at $\varepsilon_{B 1 g}=-0.18 \times 10^{-2}$, then shrinks as $\varepsilon_{B 1 g}$ becomes more negative. In order to estimate the magnitude of the strain-induced nematicity at this strain, we evaluate parameters in a Ginzburg-Landau free energy:

$$
F=\frac{\alpha \times\left(T-T_{s, 0}\right)}{2} \psi^{2}+\frac{b}{4} \psi^{4}+\frac{c}{2} \varepsilon_{B 1 g}^{2}-\lambda \varepsilon_{B 1 g} \psi .
$$

We take $\psi$ to be the splitting between the $x z$ and $y z$ orbitals at the $X$ point, which grows in an order-parameterlike fashion with cooling below $T_{s}$ and reaches $0.05 \mathrm{eV}$ as $T \rightarrow 0$ [50-52]. Numerical values for each parameter are determined from experimental data, as explained in Appendix A 9. The strain dependence of $\psi$ can then be obtained by solving $d F / d \psi=0$ under conditions of fixed strain. Doing so and evaluating at $90 \mathrm{~K}$ gives the result shown in Fig. 8(c). The maximum in the resistive anisotropy is found to occur when $\psi \approx 0.025 \mathrm{eV}$, in other words, when $\psi$ is approximately half of its $T \rightarrow 0$ value. This conclusion is robust against reasonable variation of the Ginzburg-Landau parameters. When an unstressed sample is cooled, $\psi$ reaches half its $T \rightarrow 0$ value at $\approx 80 \mathrm{~K}$ [50], and so we can conclude that resistive anisotropy is a maximum for $\psi / \psi(T \rightarrow 0) \approx 0.5$ whether $\psi$ is induced through applied strain or by allowing the sample to cool.

\section{DISCUSSION}

We first summarize our findings.

(1) The resistive anisotropy $\left(\rho_{a}-\rho_{b}\right) /\left(\rho_{a}+\rho_{b}\right)$ evolves nonmonotonically as nematicity $\psi$ grows, peaking at $\approx 7 \%$ and then decreasing [Fig. 8(a)]. Both when $\psi$ grows spontaneously with cooling and when it is induced through strain at $T \approx T_{s}$, resistive anisotropy is maximum when $|\psi|$ is about half its spontaneous $T \rightarrow 0$ value.

(2) The nematic resistive anisotropy changes sign at $T \sim 40 \mathrm{~K}$, and at low temperature, where the nematicity is fully developed, it is only $\approx-1.5 \%$ [Fig. 8(a)].

(3) At $T \approx T_{s}, \rho_{A 1 g} \equiv \frac{1}{2}\left(\rho_{a}+\rho_{b}\right)$ is a minimum when the sample is tetragonal [Fig. 6(c)].

(4) Below $\approx 60 \mathrm{~K}$ biaxial compression increases both $\rho_{a}+\rho_{b}$ [Fig. 7(a)] and $T_{c}$ [Fig. 7(b)], in opposition to the general expectation that compression increases bandwidths and weakens correlations.

This dataset places previous low-strain measurements [17-19] in context of the response over a wider strain range, over which elastoresistivity is a nontrivial function of nematicity $\psi$. It allows definitive determination of the spontaneous nematic resistive anisotropy. These results are described above, so we focus the remaining discussion on possible microscopic origins.

We first consider whether the observed elastoresistivity is a property of the mean-field nematic state. The nematic transition point at $\varepsilon_{B 1 g}=0$ and $T=T_{s}$ is a critical point of the twinning transition [see Fig. 3(b)], and the fact that elastoresistivity is particularly large in its vicinity, but shrinks quickly upon moving away from it in either temperature or strain, raises the possibility that strong elastoresistivity is a consequence of critical nematic fluctuations rather than a property of the mean-field nematic state. However, two observations argue against this possibility. One is that for $T \approx T_{s}, \rho_{A 1 g}$ is a minimum near $\varepsilon_{B 1 g}=0$ [Fig. 6(c)], whereas if critical fluctuations contributed strongly to resistivity, one would expect it to be maximum. The other is that the elastoresistivity is much stronger for strain aligned with than transverse to the principal axes of the nematicity (that is, $\left|d \rho_{B 1 g} / d \varepsilon_{B 1 g}\right| \gg\left|d \rho_{B 2 g} / d \varepsilon_{B 2 g}\right|$ ), as expected for mean-field nematic susceptibility. We therefore interpret the resistivities observed here as those of the mean-field nematic state.

The effects of biaxial strain at low temperature, like the observation that the superconducting gap magnitude correlates with $y z$ orbital weight $[21,22]$, point to an important role for the $y z$ orbital in electronic correlations. The $y z$ orbital is the only one with weight both on the $\Gamma$ and $X$ pockets, and so is thought to be the dominant contributor to $(\pi, 0)$ spin fluctuations [53]. Inelastic neutron scattering measurements have shown that the onset of nematicity correlates with stronger $(\pi, 0)$ spin fluctuations; Refs. [45,54] show that there is transfer of weight, at 
energies $\sim k_{B} T_{s}$ relevant for transport at $T \sim T_{s}$, from $(\pi, \pi)$ to $(\pi, 0)$ and/or $(0, \pi)$, while in Ref. [55] it is shown that the transfer is to $(\pi, 0)$ rather than $(0, \pi)$. At low temperatures the maximum $y z$ weight on the $\Gamma$ pocket is only $20 \%$ [22]. Biaxial compression, by weakening nematicity and increasing bandwidths, will increase this value, potentially strengthening the channel for $(\pi, 0)$ spin fluctuations and causing the increase in both resitivity and $T_{c}$.

We focus the rest of our discussion on the nonmonotonic dependence of the resistive anisotropy on both temperature and strain. We first point out that the sign change in $\rho_{a}-\rho_{b}$ occurs within the inelastic component of the resistivity. A possible explanation for a sign change in resistive anisotropy is that the inelastic and elastic components of the resistivity contribute oppositely, and balance at some temperature. At $40 \mathrm{~K}$, however, the resistivity is about 4 times the residual resistivity (based on reasonable extrapolation of the resistivity to $T \rightarrow 0$ ), so for this explanation to apply, the elastic resistive anisotropy would need to be about 4 times the inelastic resistive anisotropy, or $\sim 28 \%$. The resistive anisotropy would then grow to $\sim 28 \%$ at very low temperatures, in disagreement with the observation that it reaches only $1 \%-2 \%$.

The observed temperature dependence of the resistive anisotropy does not track thermodynamic measures of nematicity. The orthorhombicity of the unstressed lattice $[13,40,43-45]$, the anisotropy of the magnetic susceptibility [56], and the energy splitting between the $x z$ and $y z$ bands [51,52] all increase in a monotonic, order-parameter-like fashion below $T_{s}$. Several factors could cause temperature-dependent changes in resistivity. For example, in Ref. [57] it is found that shifting the relative importance of impurity versus spin fluctuation scattering can change the sign of the resistive anisotropy in ironbased superconductors. It is therefore important that this nonmonotonicity is also observed when nematicity is induced at fixed temperature, showing that it is not a temperature effect alone but intrinsic to the development of nematicity.

The importance of this observation rests on the relationship between resistive anisotropy and spin fluctuations. Spin fluctuations are found in theoretical work to dominate the resistivity at higher temperatures [53,57-61], and in optical conductivity measurements the dc resistive anisotropy is indeed found to track the scattering rate rather than the Drude weight [62]. In Ref. [53], $(\pi, 0)$ fluctuations relying on the $y z$ orbital weight were found to give $\rho_{a}>\rho_{b}$, as observed, because on the hole pocket stronger scattering of quasiparticles with $y z$ weight suppresses conduction in the $x$ direction. At lower temperatures, when spin fluctuations are weak, the precise locations of nesting-driven hot spots on the Fermi surface may be decisive in determining the sign of resistive anisotropy [27,63], making it sensitive to details, but as temperature is raised the precise nesting conditions become less important [57].

A further intuitive reason to expect $(\pi, 0)$ spin fluctuations to play a strong role in transport is that they connect the $\Gamma$ and $X$ Fermi surface pockets, providing a channel for umklapp scattering and momentum relaxation along the $k_{x}$ direction. In a clean lattice, momentum is ultimately transferred to the lattice through umklapp scattering. In systems with closed Fermi surfaces, small-angle electron-phonon scattering can transfer momentum between the electrons and phonons, but does not relax the momentum of the combined system, and so does not contribute to de resistivity. This is seen in weakly correlated metals (where the electron-phonon term is readily observable) as a modification of the usual $T^{5}$ dependence for electron-phonon resistivity to exponential, with the activation energy corresponding to a phonon that connects Fermi surfaces [64,65]. The fact that $\rho_{a}$ increases when nematicity onsets [see the inset of Fig. 8(a)], while $\rho_{b}$ decreases, is qualitatively consistent with the $(\pi, 0)$ spin fluctuations providing a mechanism for faster relaxation of transport currents along $k_{x}$.

We propose a specific mechanism for the nonmonotonic dependence of resistive anisotropy, consistent with data so far. $(\pi, 0)$ spin fluctuations, and the associated resistive anisotropy, strengthen as nematicity initially onsets and the Fermi velocity on the $y z$ sections of Fermi surface is reduced. These fluctuations then weaken as the nematicity grows further and suppresses the $y z$ orbital weight on the hole pocket, cutting off this fluctuation channel. This is a proposal and a point for further investigation; the relative contributions of spin fluctuation strength and nematicitydriven changes in Fermi surface shape to resistive anisotropy need to be determined. However, direct measurement of spin fluctuations under tunable lattice strain, through inelastic neutron scattering, would be a very challenging experiment. It is nevertheless an important route to attempt because it could provide a direct test of a major class of theories of the nematicity of $\mathrm{FeSe}$, in which it is proposed to be driven by the increase in phase space that it allows for spin fluctuations [66-68]. The potential challenge to these theories, if the nonmonotonic resistive anisotropy observed here indeed correlates with nonmonotonic spin fluctuation strength, is to explain why the nematicity grows well past the point where it maximizes spin fluctuation strength.

Regardless of how that path of inquiry develops, we anticipate that the strain-tuning capabilities demonstrated here will allow resolution of the separate orbital contributions to the electronic properties of FeSe and theories of the nematicity of FeSe to be tested.

The data that support the findings of this study are openly available from the Max Planck Digital Library [69]. 


\section{ACKNOWLEDGMENTS}

We thank Hiroshi Kontani, Andreas Kreisel, Kazuhiko Kuroki, Seiichiro Onari, Sahana Rößler, Jörg Schmalian, Roser Valentí, Matthew Watson, and Steffen Wirth for useful discussions. S. H. and T. S. thank S. Kasahara, Y. Matsuda, K. Matsuura, and Y. Mizukami for earlystage collaboration on sample growth. We thank the Max Planck Society for financial support. C. W. H., A. P. M., and C. T. acknowledge support by the DFG (DE) through the Collaborative Research Centre SFB 1143 (Projects C09 and A04). C. T. acknowledges support by the DFG (DE) through the Cluster of Excellence on Complexity and Topology in Quantum Matter ct.qmat (EXC 2147). Work in Japan was supported by Grants-in-Aid for Scientific Research (KAKENHI) (No. JP19H00649 and No. JP18H05227), and Grant-in-Aid for Scientific Research on innovative areas "Quantum Liquid Crystals" (No. JP19H05824 and No. JP20H05162) from Japan Society for the Promotion of Science (JSPS).

Note added in proof.-Recent work reports uniaxial stress measurements on free-standing samples of FeSe. The elastoresistivity for stress applied along an $\mathrm{Fe}-\mathrm{Fe}$ bond direction, inducing $B_{1 g}$ strain in our notation, is found to change sign at $70 \mathrm{~K}[70]$.

\section{APPENDIX: ADDITIONAL DATA}

\section{Montgomery conversion}

To measure the resistivity $\rho_{x x}$ parallel to the direction of applied stress, we used a four-point configuration with barshaped samples. To measure $\rho_{y y}$, in principle bar-shaped samples could be mounted perpendicular to the stress axis. However, the narrowness of the platform neck makes this inconvenient; to avoid excessively loading the stress cell, we cannot substantially widen this neck. Therefore, a Montgomery configuration was used to determine $\rho_{x x}$ and $\rho_{y y}$ simultaneously. These methods are reviewed in Ref. [71].

We follow the analysis method presented in Refs. [7275]. The sample is a rectangle with length $L_{1}^{\prime}$, width $L_{2}^{\prime}$, and thickness $L_{3}^{\prime}$, and resistivities along these lengths $\rho_{1}, \rho_{2}$, and $\rho_{3}$. Two resistances are measured: $R_{1}$ is the voltage measured across two corners separated by length $L_{1}^{\prime}$ divided by current applied across the other two corners, and $R_{2}$ is the equivalent resistance measured along length $L_{2}^{\prime}$. The sample can be transformed into an equivalent sample with isotropic resistivity $\rho$ and dimensions $L_{1}, L_{2}$, and $L_{3}$, where the definition of equivalence is that the same resistances would be measured as on the original sample.

The resistivity of this isotropic equivalent is given by

$$
\rho=H_{1} t_{\mathrm{eff}} R_{1}=H_{2} t_{\mathrm{eff}} R_{2},
$$

where $t_{\text {eff }}$ is an effective sample thickness. In the limit $L_{3} \ll\left(L_{1} L_{2}\right)^{1 / 2}$, which applies here, $t_{\mathrm{eff}} \approx L_{3} . H_{1}$ is a function only of the geometry of the sample:

$$
\frac{1}{H_{1}}=\frac{8}{\pi} \sum_{n=0}^{\infty}\left\{(2 n+1) \sinh \left[\pi(2 n+1) \frac{L_{2}}{L_{1}}\right]\right\}^{-1} .
$$

$H_{2}$ is obtained by switching $L_{1}$ and $L_{2}$ in this expression. This series converges rapidly. Using the constraint from Eq. (A1) that $H_{1} R_{1}=H_{2} R_{2}$, we compute the first few terms in order to solve for $L_{2} / L_{1}$.

The transformation from anisotropic to isotropic equivalent solid is given by:

$$
\begin{aligned}
L_{i} & =L_{i}^{\prime}\left(\frac{\rho_{i}}{\rho}\right)^{\frac{1}{2}}, \\
\rho^{3} & =\rho_{1} \rho_{2} \rho_{3} .
\end{aligned}
$$

Using these expressions, from Eq. (A1) one can quickly obtain:

$$
\left(\rho_{1} \rho_{2}\right)^{1 / 2}=H_{1} L_{3}^{\prime} R_{1} .
$$

They also give a relationship between $\rho_{1}$ and $\rho_{2}$ :

$$
\frac{\rho_{2}}{\rho_{1}}=\left(\frac{L_{1}^{\prime}}{L_{2}^{\prime}}\right)^{2}\left(\frac{L_{2}}{L_{1}}\right)^{2}
$$

which yields

$$
\rho_{1}=H_{1} L_{3}^{\prime} R_{1} \frac{L_{2}^{\prime}}{L_{1}^{\prime}} \frac{L_{1}}{L_{2}} .
$$

The sample dimensions vary slightly as strain is applied. We take $L_{1}^{\prime}=\left(1+\varepsilon_{x x}\right) \times L_{1,0}^{\prime}$, where $L_{1,0}^{\prime}$ is the unstrained sample length, and $L_{2}^{\prime}=\left(1-\nu \varepsilon_{x x}\right) \times L_{2,0}^{\prime} \cdot \varepsilon_{x x}$ is the strain along the length of the platform. As a matter of formal correctness, we also include an effect of applied strain on the $c$-axis lattice constant: we take $L_{3}^{\prime}=-0.7 L_{3,0}^{\prime} \varepsilon_{A 1 g}$. However, the effect is negligible and in practice it would serve just as well here to take $L_{3}^{\prime}$ to be constant.

\section{Strain transmission}

When the epoxy and sample layers are both thin and the epoxy elastic moduli are low, strain transfer to the sample can be characterized to good accuracy by a strain transmission length $\lambda$, given by $\lambda=(c t d / G)^{1 / 2}$, where $c$ is the relevant elastic modulus of the sample, $t$ the sample thickness, $d$ the epoxy thickness, and $G$ the epoxy shear modulus [76]. Under the conditions that the $c$-axis strain in the sample is unconstrained while the transverse strain is fixed, $c=$ $c_{11}-c_{13}^{2} / c_{33}$ [34]. Even though the Young's modulus of FeSe becomes nearly zero for $T \approx T_{s}$ [7], $c$ remains substantial, at $\approx 40 \mathrm{GPa}$ based on the elastic moduli reported in Refs. $[39,77,78]$. Physically, this means that the lattice remains stiff against biaxial compression, even as it becomes soft against orthorhombic distortion. To determine $d$, a focused ion beam was used to slice through some of the 
TABLE II. Sample parameters: length, width, thickness, separation $l_{\text {contact }}$ of the voltage contacts, and the residual resistivity ratio (RRR) $\rho(300 \mathrm{~K}) / \rho(12 \mathrm{~K})$. Note that at $12 \mathrm{~K}$ there is still strong inelastic scattering.

\begin{tabular}{lrcccc}
\hline \hline Sample & $l(\mu \mathrm{m})$ & $w(\mu \mathrm{m})$ & $t(\mu \mathrm{m})$ & $l_{\text {contact }}(\mu \mathrm{m})$ & RRR \\
\hline A & 2370 & 280 & 31 & 970 & 26 \\
B & 1150 & 230 & 10 & 630 & 22 \\
C & 434 & 425 & $\approx 10$ & & \\
\hline \hline
\end{tabular}

samples at a few points; an example of a cross section through sample B is shown in Fig. 1(d). $d$ was found to be 5-10 $\mu \mathrm{m}$. To estimate $G$ we take the Young's modulus of Stycast 1266, reported in Ref. [79], and assume a Poisson ratio of 0.3 , which gives $G=1.6 \mathrm{GPa}$ at low temperature.

Samples A and B are both long, ensuring good coupling of longitudinal strain to the platform, and so the key question is of their width in comparison with $\lambda$. For samples much narrower than $\lambda$, the transverse strain is the longitudinal strain multiplied by the sample's Poisson ratio, while for samples much wider than $\lambda$, it is the longitudinal strain multiplied by the platform's Poisson ratio. For FeSe this is an important distinction because its Poisson ratio for $T \sim T_{s}$ is close to 1 , while that of titanium is 0.32 . We find that both samples A and B are wide enough to ensure good locking of transverse strain to the platform. In particular, sample $\mathrm{A}$ is $31 \mu \mathrm{m}$ thick, yielding $\lambda \approx 60 \mu \mathrm{m}$, while its width is $280 \mu \mathrm{m}$. Sample B is $10 \mu \mathrm{m}$ thick, yielding $\lambda \approx 40 \mu \mathrm{m}$, and $230 \mu \mathrm{m}$ wide. Complete sample dimensions are shown in Table II.

\section{Elastic moduli}

Reference [39] gives elastic moduli of FeSe at $T \approx T_{s}$ : $c_{11} \approx c_{12} \approx 50 \mathrm{GPa}, c_{33} \approx 40 \mathrm{GPa}$, and $c_{13} \approx 20 \mathrm{GPa}$. Under conditions of hydrostatic pressure, $\sigma / \varepsilon_{x x}=\left(c_{11} c_{33}+\right.$ $\left.c_{12} c_{33}-2 c_{13}^{2}\right) /\left(c_{33}-c_{13}\right)$, where $\sigma$ is the applied stress, and $\varepsilon_{z z} / \varepsilon_{x x}=\left(c_{11}+c_{12}-2 c_{13}\right) /\left(c_{33}-c_{13}\right)$. Under conditions of in-plane biaxial stress, where $\sigma_{x x}=\sigma_{y y}$ and $\sigma_{z z}=0, \sigma_{x x} / \varepsilon_{x x}=c_{11}+c_{12}-2 c_{13}^{2} / c_{33}$ and $\varepsilon_{z z} / \varepsilon_{x x}=$ $-2 c_{13} / c_{33}$.

\section{Plastic deformation of the platform}

Sample B was driven to high compressions, and the platform deformed plastically when the displacement $D$ applied to it exceeded $\approx 7 \mu \mathrm{m}$, causing the strain in the neck to exceed the elastic limit of the platform material, $\approx 2 \times 10^{-3}$. Data from sample B were taken in the following order. (1) Strain ramps were performed at $T \approx T_{s}$ up to modest strains. (2) Temperature ramps were performed at constant strain, incrementing the strain at $103.7 \mathrm{~K}$, and moving gradually to high compressions. (3) Further strain ramps were performed at high compression. Data from these three sets are plotted against $D$ in Figs. 9(a) and 9(b). There is low hysteresis within each strain-ramp dataset, and
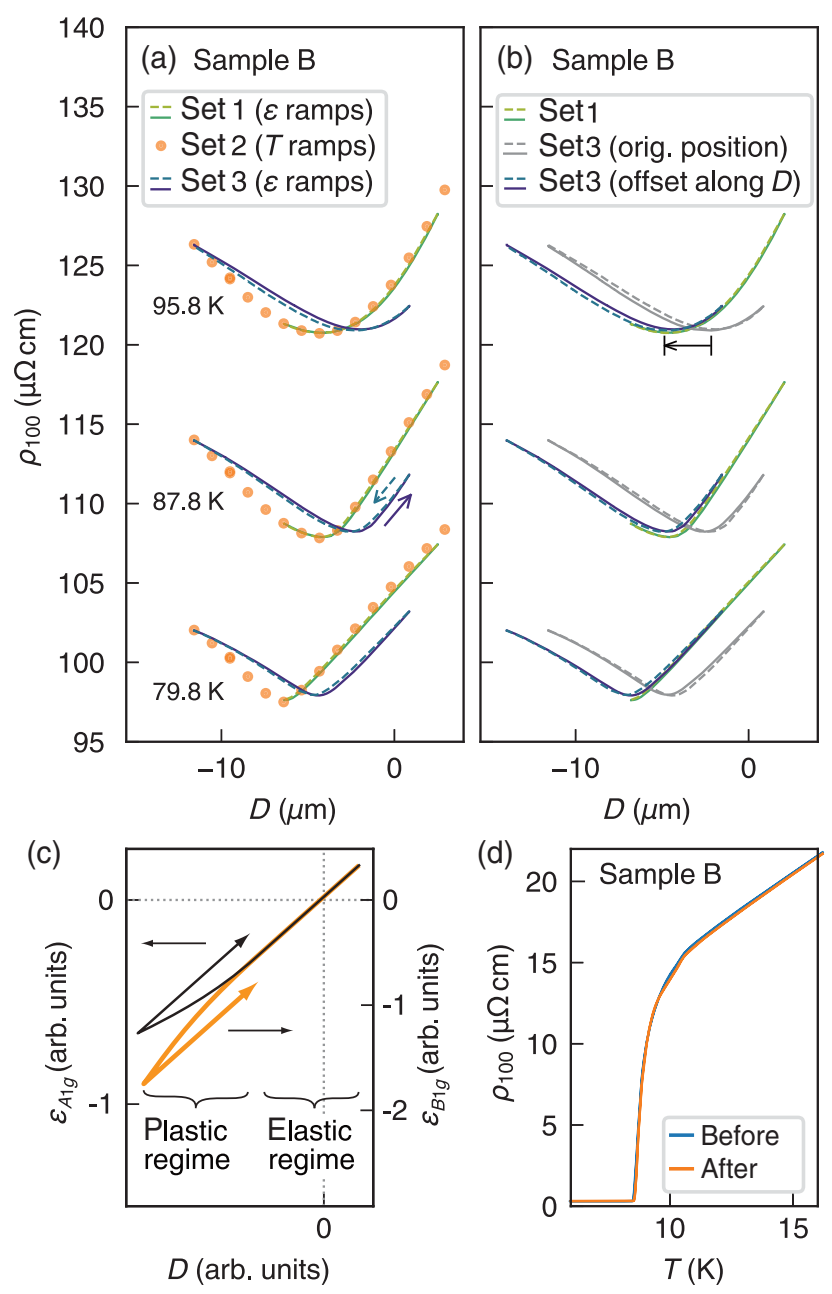

FIG. 9. Plastic deformation of the platform. (a) $\rho_{100}$ of sample B versus displacement $D$ applied to the platform. The datasets were taken in the following order. (1) Strain ramps at fixed temperature. (2) Temperature ramps at fixed strain. (3) Strain ramps at higher compression. The offset between datasets (1) and (3) is due to plastic deformation of the platform that occurred over the course of the temperature ramps. (b) When data from set 3 are offset along the $D$ axis, the match with dataset 1 is excellent. (c) Schematic illustration of the process of plastic deformation. (d) Low-temperature resistivity measured before and after the platform plastic deformation. To compare datasets where $T_{c}$ was the same, the before data are taken at $D=-1.6 \mu \mathrm{m}$ and the after data at $D=0.8 \mu \mathrm{m}$.

the two strain-ramp datasets match closely except for an offset along the $D$ axis. The temperature-ramp data bridge this offset smoothly. We conclude that the platform deformation was essentially elastic within each strain-ramp dataset, and that the offset between them is due to plastic deformation caused by the large change in applied strain over the course of the temperature ramps.

Figure 9(c) shows a schematic illustration of the expected form of the plastic deformation. Initially, when the platform deformation is elastic, $\varepsilon_{A 1 g}$ and $\varepsilon_{B 1 g}$ are linear in $D: \varepsilon_{B 1 g}=$ $0.66 D / l_{\text {eff }}$ and $\varepsilon_{A 1 g}=0.34 D / l_{\text {eff }}$ (where $l_{\text {eff }}$ is the effective length of the platform). Beyond its elastic limit, the platform 


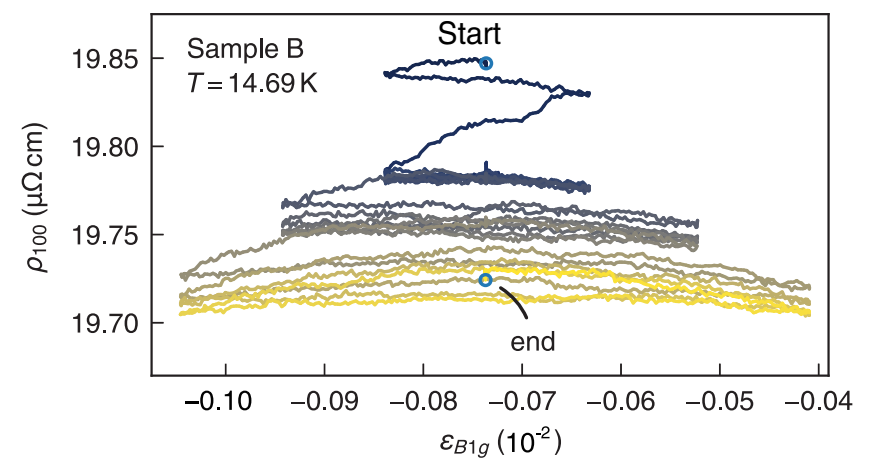

FIG. 10. Annealing twin boundaries out of the sample by ramping the applied strain.

material resists further volume compression by flowing plastically outward: $\varepsilon_{B 1 g}$ starts to vary more steeply with $D$, and $\varepsilon_{A 1 g}$ less steeply. When the direction of the applied displacement is reversed, the platform deformation is again elastic over some range, but for a given $D \varepsilon_{B 1 g}$ is larger and $\varepsilon_{A 1 g}$ smaller than before.

That the sample deformation remained elastic even as the platform deformed plastically is shown in Fig. 9(d), in which low-temperature data from before and after the plastic deformation, taken at strains where $T_{c}$ is the same, are plotted together. The residual resistivity is unchanged.

The sign of the offset between the pre- and post-plasticdeformation data shows that $\rho_{100}$ is controlled dominantly by $\varepsilon_{B 1 g}$, rather than $\varepsilon_{A 1 g}$. The fact that a horizontal displacement works so well to match the pre- and postplastic-deformation data shows that the effect of $\varepsilon_{A 1 g}$ on $\rho_{100}$ is small; if it were strong, then it would have to be finely balanced, over a wide temperature range, with that of $\varepsilon_{B 1 g}$ for the net effect to be so neatly a horizontal shift of the $\rho(D)$ curves. Furthermore, the data of Fig. 2 show directly that the dependence of $\rho$ on $\varepsilon_{A 1 g}$ is weak.

In Figs. 5(a) and 5(c), to account for this plastic platform deformation data from the high-strain strain ramps are offset by $\Delta \varepsilon_{B 1 g}=-0.072 \times 10^{-2}$. Because this deformation occurred gradually over the course of the temperature ramps, for $\varepsilon_{B 1 g}<-0.11 \times 10^{-2}$ each individual temperature ramp is offset along the $\varepsilon_{B 1 g}$ axis to match the resistivity at $103.7 \mathrm{~K}$ with that from the strain ramps.

\section{Annealing twin boundaries}

In Fig. 10 we show results of a twin boundary annealing experiment. Sample B was cooled from above $T_{s}$ to $14.69 \mathrm{~K}$ at a fixed strain. The strain was then ramped back and forth. Over the first few cycles of strain ramping, the sample resistance falls, but then settles at a lower value. When the strain-ramp amplitude is then increased, the decrease in resistance resumes, and then the resistance settles at a yet lower value. This behavior shows that twin boundaries can be partially annealed out of the sample
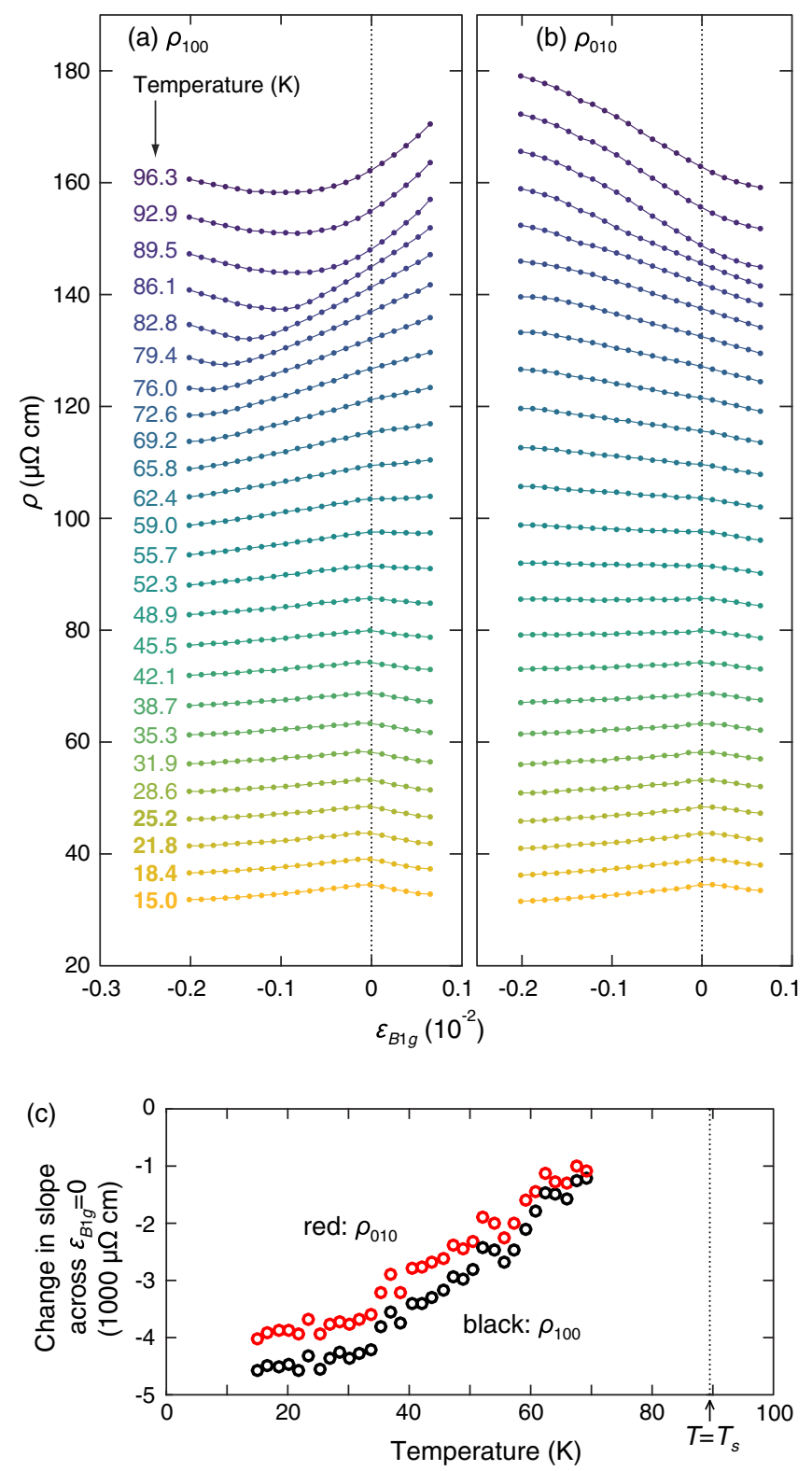

FIG. 11. (a),(b) Temperature-ramp data from sample C. Panel (a) shows $\rho_{100}$ and (b) $\rho_{010}$. (c) Change in slope $d \rho / d \varepsilon_{B 1 g}$ across $\varepsilon_{B 1 g}=0$. This quantity is proportional to the twin boundary contribution to sample resistivity at $\varepsilon_{B 1 g}=0$.

through strain ramps, and confirms that the peaked form of the resistance in $T$-ramp data, shown in Fig. 5, is due to twin boundaries.

\section{6. $T$-ramp data from sample $\mathrm{C}$, and twin boundary resistivity}

Temperature-ramp data from sample $\mathrm{C}$ are shown in Fig. 11. At low temperatures, the cusp in $\rho\left(\varepsilon_{B 1 g}\right)$ due to the maximum in domain wall density is visible in both $\rho_{100}$ and $\rho_{010}$. Its location differs slightly in the two measurements, possibly because in the Montgomery configuration measurements of $\rho_{100}$ and $\rho_{010}$ do not probe precisely the 


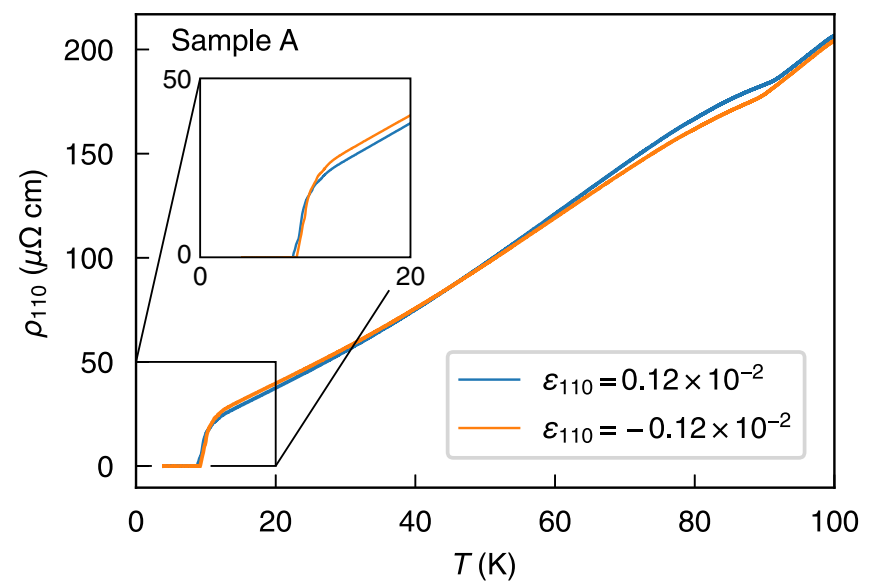

FIG. 12. $\rho_{110}$ versus $T$ of sample A over a wide temperature range.

same area of the sample. We take $\varepsilon_{B 1 g}=0$ as the average of the cusp locations in $\rho_{100}$ and $\rho_{010}$.

In Fig. 11(c), we show the change in slope $d \rho / d \varepsilon_{B 1 g}$ across the cusp at $\varepsilon_{B 1 g}=0$ versus temperature. This quantity is proportional to the twin boundary contribution to sample resistivity at $\varepsilon_{B 1 g}=0$. The twin boundary resistivity is seen to be nearly $T$ independent up to $\sim 30 \mathrm{~K}$, and then to decrease. Note that this is the twin boundary resistivity when the sample is cooled from above $T_{s}$ at $\varepsilon_{B 1 g}=0$; when it is brought to $\varepsilon_{B 1 g}=0$ by ramping strain at constant temperature, the twin boundary density is lower.

In Figs. 7(a) and 8(a), elastoresistivities normalized by $\rho_{a}+\rho_{b}$ are shown. For this normalization we subtracted off an estimated twin boundary resistivity, $\rho_{\mathrm{TB}}(T)$; for example, in Fig. 8(a) the quantity that is plotted is $\rho_{a}-\rho_{b}$, determined by the underlying slopes method described in the text, divided by $\rho_{100}\left(\varepsilon_{B 1 g}=0\right)+\rho_{010}\left(\varepsilon_{B 1 g}=0\right)-$ $2 \rho_{\mathrm{TB}}(T)$. Based on the illustration in Fig. 5(c), we estimate $\rho_{\mathrm{TB}}(T \rightarrow 0)=3 \mu \Omega \mathrm{cm}$. We take $\rho_{\mathrm{TB}}=\rho_{\mathrm{TB}}(T \rightarrow 0) \times$ $\left[1-\left(T / T_{s}\right)^{2}\right]$. This form overestimates somewhat the true twin boundary resistance as $T$ approaches $T_{s}$; however, the effect is tiny.

\section{Elastoresistivity of sample A}

Figure 12 shows the elastoresistivity of sample A over a wide temperature range. The behavior qualitatively matches the $A_{1 g}$ elastoresistivity determined from sample $\mathrm{C}$ and plotted in Fig. 7(a): at higher temperatures, compression causes a decrease in resistivity, and at lower temperatures an increase. The sign of the response changes at $T \approx 45 \mathrm{~K}$, against $60 \mathrm{~K}$ for the $A_{1 g}$ elastoresistivity of sample $\mathrm{C}$. The measured resistivity of sample A will also be affected by the $B_{2 g}$ elastoresistivity; however, because this is transverse to the nematic axes, it is not expected to be large, and the qualitative agreement with the $A_{1 g}$ elastoresistivity suggests that it is indeed much smaller than the

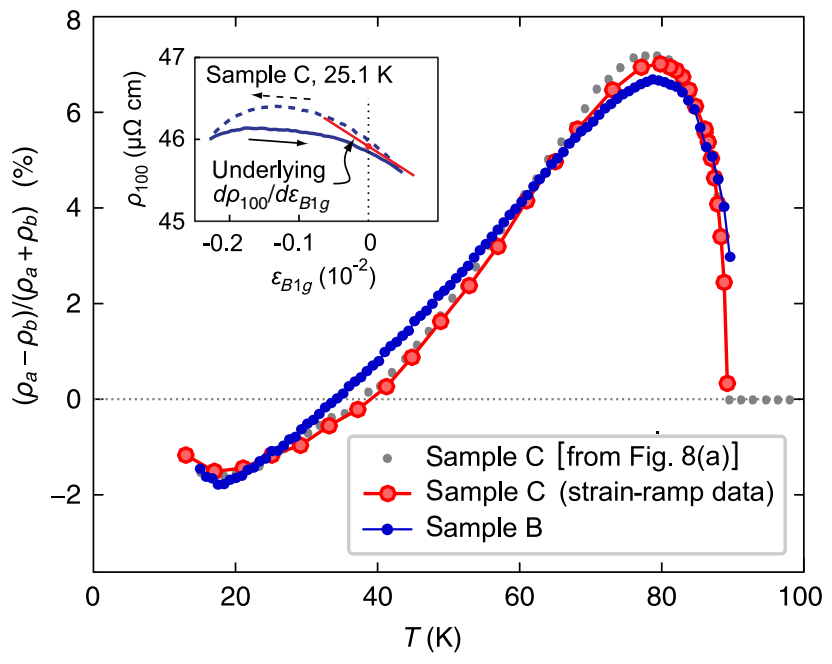

FIG. 13. Nematic resistivity anisotropy $\left(\rho_{a}-\rho_{b}\right) /\left(\rho_{a}+\rho_{b}\right)$ of sample $\mathrm{C}$, derived from the strain-ramp data shown in Fig. 6(a). This determination is based on extraction of equilibrium slopes $d \rho / d \varepsilon_{B 1 g}$ at $\varepsilon_{B 1 g}=0$, obtained by averaging the observed increasing- $\varepsilon$ and decreasing- $\varepsilon$ slopes at $\varepsilon_{B 1 g}=0$, as shown in the inset.

$A_{1 g}$ elastoresistivity. Note also that $T_{c}$ increases with compression, as observed in sample B [Fig. 7(b)].

\section{Additional derivations of the nematic resistive anisotropy}

Above, we presented a determination of the nematic resistive anisotropy for $T<T_{s}$ based on sample $\mathrm{C}$ temperature-ramp data, in which the twin distribution can be assumed to be in near equilibrium with the applied strain. Here, we analyze strain-ramp data. As described above, the determination of nematic resistive anisotropy depends on extraction of underlying slopes $d \rho_{100} / d \varepsilon_{B 1 g}$ and $d \rho_{010} / d \varepsilon_{B 1 g}$ at $\varepsilon_{B 1 g}=0$, that is, excluding the effect of twin boundaries. In the strain ramps, the density and location of twin boundaries lags the applied strain, and we therefore obtain these slopes by averaging the observed slopes from the increasing-strain and decreasing-strain ramps, as illustrated in the inset of Fig. 13. Applying Eq. (6) yields the nematic resistive anisotropy plotted in Fig. 13. The close agreement with $T$-ramp data shows that the twin boundary resistance has been properly excluded. Note that, because the twin boundary density is lower in strain-ramp than temperature-ramp data, we do not subtract off a twin boundary contribution to $\rho_{a}+\rho_{b}$ for normalization.

Also shown in Fig. 13 is the resistivity anisotropy determined from sample B. For sample B, only $\rho_{100}$ was measured. Evaluating Eq. (1) at $f=0.5$ yields

$$
\rho_{a}-\rho_{b}=2 \varepsilon_{s}\left(\frac{d \rho_{100}}{d \varepsilon_{B 1 g}}-\frac{1-\nu}{1+\nu} \frac{d \rho_{A 1 g}}{d \varepsilon_{A 1 g}}\right) .
$$


$d \rho_{A 1 g} / d \varepsilon_{A 1 g}$ must be taken from data from sample $\mathrm{C}$ [see Fig. 7(a)]; the data plotted in Fig. 13 include this correction. For the normalization our estimate for twin boundary resistivity is subtracted (see Appendix A 6).

\section{Ginzburg-Landau parameters}

In the Ginzburg-Landau free energy [Eq. (7)], the strain is the $B_{1 g}$ strain, for which the elastic constant $c$ is $c_{11}-c_{12}$. This elastic constant must be evaluated without the influence of nematic susceptibility. Reference [39] finds $c_{11} \approx 80 \mathrm{GPa}$ at $T \approx 250 \mathrm{~K}$, and electronic structure calculations give $c_{11}=95 \mathrm{GPa}$ [80]. We take the estimate $c=c_{11}-c_{12}=60 \mathrm{GPa}$. The structural strain is obtained by noting that $d F / d \varepsilon=0$ at $\varepsilon=\varepsilon_{s}$, which gives $\varepsilon_{s}=(\lambda / c) \psi$. Although the Ginzburg-Landau formalism only applies, strictly, very near to $T_{s}$, we evaluate parameters at considerably lower temperature in order to obtain approximate evaluations of the coefficients. $\varepsilon_{s} \rightarrow 0.27 \times$ $10^{-2}$ as $T \rightarrow 0$ [40], yielding a value for the coupling constant: $\lambda \approx 3.2 \mathrm{GPa} / \mathrm{eV}$. As shown in Fig. 8(b), a fit to elastoresistivity data yields a bare nematic transition temperature $T_{s, 0}=60.7 \mathrm{~K}$; we take $T_{s, 0}=60 \mathrm{~K}$. $T_{s}$ is defined by the relationship

$$
\frac{\lambda^{2}}{c}-\alpha \times\left(T_{s}-T_{s, 0}\right)=0 .
$$

Taking $T_{s}=90 \mathrm{~K}$ yields $\alpha=0.0057 \mathrm{GPa} / \mathrm{eV}^{2} \mathrm{~K}$. Finally, we evaluate $b$ from the observation that $\psi$ reaches half its $T \rightarrow 0$, or $0.025 \mathrm{eV}$, value at $T \approx 0.9 T_{s}$ [50], which gives $b=82 \mathrm{GPa} / \mathrm{eV}^{4}$.

[1] S. Licciardello, J. Buhot, J. Lu, J. Ayres, S. Kasahara, Y. Matsuda, T. Shibauchi, and N.E. Hussey, Electrical Resistivity Across a Nematic Quantum Critical Point, Nature (London) 567, 213 (2019).

[2] S. Lederer, Y. Schattner, E. Berg, and S. A. Kivelson, Enhancement of Superconductivity Near a Nematic Quantum Critical Point, Phys. Rev. Lett. 114, 097001 (2015).

[3] H. Murayama, Y. Sato, R. Kurihara, S. Kasahara, Y. Mizukami, Y. Kasahara, H. Uchiyama, A. Yamamoto, E.-G. Moon, J. Cai, J. Freyermuth, M. Greven, T. Shibauchi, and Y. Matsuda, Diagonal Nematicity in the Pseudogap Phase of $\mathrm{HgBa}_{2} \mathrm{CuO}_{4+\delta}$, Nat. Commun. 10, 3282 (2019).

[4] R. M. Fernandes, A. V. Chubukov, J. Knolle, I. Eremin, and J. Schmalian, Preemptive Nematic Order, Pseudogap, and Orbital Order in the Iron Pnictides, Phys. Rev. B 85, 024534 (2012).

[5] R. M. Fernandes, A. V. Chubukov, and J. Schmalian, What Drives Nematic Order in Iron-Based Superconductors?, Nat. Phys. 10, 97 (2014).

[6] R. M. Fernandes, A. E. Böhmer, C. Meingast, and J. Schmalian, Scaling between Magnetic and Lattice Fluctuations in Iron Pnictide Superconductors, Phys. Rev. Lett. 111, 137001 (2013).
[7] A. E. Böhmer, T. Arai, F. Hardy, T. Hattori, T. Iye, T. Wolf, H. v. Löhneysen, K. Ishida, and C. Meingast, Origin of the Tetragonal-to-Orthorhombic Phase Transition in FeSe: A Combined Thermodynamic and NMR Study of Nematicity, Phys. Rev. Lett. 114, 027001 (2015).

[8] S.-H. Baek, D. V. Efremov, J. M. Ok, J. S. Kim, J. van den Brink, and B. Büchner, Orbital-Driven Nematicity in FeSe, Nat. Mater. 14, 210 (2015).

[9] T. Terashima, N. Kikugawa, S. Kasahara, T. Watashige, T. Shibauchi, Y. Matsuda, T. Wolf, A. E. Böhmer, F. Hardy, C. Meingast, H. von Lhneysen, and S. Uji, PressureInduced Antiferromagnetic Transition and Phase Diagram in FeSe, J. Phys. Soc. Jpn. 84, 063701 (2015).

[10] A. E. Böhmer, K. Kothapalli, W. T. Jayasekara, J. M. Wilde, B. Li, A. Sapkota, B. G. Ueland, P. Das, Y. Xiao, W. Bi, J. Zhao, E. E. Alp, S. L. Budko, P. C. Canfield, A. I. Goldman, and A. Kreyssig, Distinct Pressure Evolution of Coupled Nematic and Magnetic Orders in FeSe, Phys. Rev. B 100, 064515 (2019).

[11] H. Pfau, S. D. Chen, M. Yi, M. Hashimoto, C. R. Rotundu, J. C. Palmstrom, T. Chen, P.-C. Dai, J. Straquadine, A. Hristov, R. J. Birgeneau, I. R. Fisher, D. Lu, and Z.-X. Shen, Momentum Dependence of the Nematic Order Parameter in Iron-Based Superconductors, Phys. Rev. Lett. 123, 066402 (2019).

[12] M. Yi, H. Pfau, Y. Zhang, Y. He, H. Wu, T. Chen, Z. R. Ye, M. Hashimoto, R. Yu, Q. Si, D.-H. Lee, P. Dai, Z.-X. Shen, D. H. Lu, and R. J. Birgeneau, Nematic Energy Scale and the Missing Electron Pocket in FeSe, Phys. Rev. X 9 , 041049 (2019).

[13] A. E. Böhmer, F. Hardy, F. Eilers, D. Ernst, P. Adelmann, P. Schweiss, T. Wolf, and C. Meingast, Lack of Coupling between Superconductivity and Orthorhombic Distortion in Stoichiometric Single-Crystalline FeSe, Phys. Rev. B 87, 180505(R) (2013).

[14] S. Kasahara, T. Watashige, T. Hanaguri, Y. Kohsaka, T. Yamashita, Y. Shimoyama, Y. Mizukami, R. Endo, H. Ikeda, K. Aoyama, T. Terashima, S. Uji, T. Wolf, H. von Löhneysen, T. Shibauchi, and Y. Matsuda, Field-Induced Superconducting Phase of FeSe in the BCS-BEC Cross-Over, Proc. Natl. Acad. Sci. U.S.A. 111, 16309 (2014).

[15] J.-H. Chu, H.-H. Kuo, J. G. Analytis, and I. R. Fisher, Divergent Nematic Susceptibility in an Iron Arsenide Superconductor, Science 337, 710 (2012).

[16] H.-H. Kuo, J.-H. Chu, J. C. Palmstrom, S. A. Kivelson, and I. R. Fisher, Ubiquitous Signatures of Nematic Quantum Criticality in Optimally Doped Fe-Based Superconductors, Science 352, 958 (2016).

[17] M. D. Watson, T. K. Kim, A. A. Haghighirad, N. R. Davies, A. McCollam, A. Narayanan, S. F. Blake, Y. L. Chen, S. Ghannadzadeh, A. J. Schofield, M. Hoesch, C. Meingast, T. Wolf, and A. I. Coldea, Emergence of the Nematic Electronic State in FeSe, Phys. Rev. B 91, 155106 (2015).

[18] S. Hosoi, K. Matsuura, K. Ishida, H. Wang, Y. Mizukami, T. Watashige, S. Kasahara, Y. Matsuda, and T. Shibauchi, Nematic Quantum Critical Point without Magnetism in FeSe $_{1-x} \mathrm{~S}_{x}$ Superconductors, Proc. Natl. Acad. Sci. U.S.A. 113, 8139 (2016).

[19] M. A. Tanatar, A. E. Böhmer, E. I. Timmons, M. Schütt, G. Drachuck, V. Taufour, K. Kothapalli, A. Kreyssig, 
S. L. Bud'ko, P. C. Canfield, R. M. Fernandes, and R. Prozorov, Origin of the Resistivity Anisotropy in the Nematic Phase of FeSe, Phys. Rev. Lett. 117, 127001 (2016).

[20] L. de' Medici, G. Giovannetti, and M. Capone, Selective Mott Physics as a Key to Iron Superconductors, Phys. Rev. Lett. 112, 177001 (2014).

[21] P. O. Sprau, A. Kostin, A. Kreisel, A. E. Böhmer, V. Taufour, P. C. Canfield, S. Mukherjee, P. J. Hirschfeld, B. M. Andersen, and J.C. Davis, Discovery of OrbitalSelective Cooper Pairing in FeSe, Science 357, 75 (2017).

[22] L. C. Rhodes, M. D. Watson, A. A. Haghighirad, D. V. Evtushinsky, M. Eschrig, and T. K. Kim, Scaling of the Superconducting Gap with Orbital Character in FeSe, Phys. Rev. B 98, 180503(R) (2018).

[23] T. Shibauchi, T. Hanaguri, and Y. Matsuda, Exotic Superconducting States in FeSe-Based Materials, J. Phys. Soc. Jpn. 89, 102002 (2020).

[24] J.-H. Chu, J. G. Analytis, K. De Greve, P. L. McMahon, Z. Islam, Y. Yamamoto, and I. R. Fisher, In-Plane Resistivity Anisotropy in an Underdoped Iron Arsenide Superconductor, Science 329, 824 (2010).

[25] H.-H. Kuo, J. G. Analytis, J.-H. Chu, R. M. Fernandes, J. Schmalian, and I. R. Fisher, Magnetoelastically Coupled Structural, Magnetic, and Superconducting Order Parameters in $\mathrm{BaFe}_{2}\left(\mathrm{As}_{1-x} \mathrm{P}_{x}\right)_{2}$, Phys. Rev. B 86, 134507 (2012).

[26] E. C. Blomberg, A. Kreyssig, M. A. Tanatar, R. M. Fernandes, M. G. Kim, A. Thaler, J. Schmalian, S. L. Bud'ko, P. C. Canfield, A. I. Goldman, and R. Prozorov, Effect of Tensile Stress on the In-Plane Resistivity Anisotropy in $\mathrm{BaFe}_{2} \mathrm{As}_{2}$, Phys. Rev. B 85, 144509 (2012).

[27] E. C. Blomberg, M. A. Tanatar, R. M. Fernandes, I. I. Mazin, B. Shen, H.-H. Wen, M. D. Johannes, J. Schmalian, and R. Prozorov, Sign-Reversal of the In-Plane Resistivity Anisotropy in Hole-Doped Iron Pnictides, Nat. Commun. 4, 1914 (2013).

[28] X.-Y. Lu, J. T. Park, R. Zhang, H.-Q. Luo, A. H. Nevidomskyy, Q.-M. Si, and P.-C. Dai, Nematic Spin Correlations in the Tetragonal State of Uniaxial-Strained $\mathrm{BaFe}_{2-x} \mathrm{Ni}_{x} \mathrm{As}_{2}$, Science 345, 657 (2014).

[29] A. V. Maharaj, E. W. Rosenberg, A. T. Hristov, E. Berg, R. M. Fernandes, I. R. Fisher, and S. A. Kivelson, Transverse Fields to Tune an Ising-Nematic Quantum Phase Transition, Proc. Natl. Acad. Sci. U.S.A. 114, 13430 (2017).

[30] T. Terashima, N. Kikugawa, A. Kiswandhi, E.-S. Choi, J. S. Brooks, S. Kasahara, T. Watashige, H. Ikeda, T. Shibauchi, Y. Matsuda, T. Wolf, A. E. Böhmer, F. Hardy, C. Meingast, H. v. Löhneysen, M. T. Suzuki, R. Arita, and S. Uji, Anomalous Fermi Surface in FeSe Seen by Shubnikov-de Haas Oscillation Measurements, Phys. Rev. B 90, 144517 (2014).

[31] M. D. Watson, T. Yamashita, S. Kasahara, W. Knafo, M. Nardone, J. Béard, F. Hardy, A. McCollam, A. Narayanan, S. F. Blake, T. Wolf, A. A. Haghighirad, C. Meingast, A. J. Schofield, H. v. Löhneysen, Y. Matsuda, A. I. Coldea, and T. Shibauchi, Dichotomy between the Hole and Electron Behavior in Multiband Superconductor FeSe Probed by Ultrahigh Magnetic Fields, Phys. Rev. Lett. 115, 027006 (2015).
[32] M. D. Watson, A. A. Haghighirad, L. C. Rhodes, M. Hoesch, and T. K. Kim, Electronic Anisotropies Revealed by Detwinned Angle-Resolved Photo-Emission Spectroscopy Measurements of FeSe, New J. Phys. 19, 103021 (2017).

[33] L. C. Rhodes, M. D. Watson, A. A. Haghigirad, D. V. Evtushinsky, and T. K. Kim, Revealing the Single Electron Pocket of FeSe in a Single Orthorhombic Domain, Phys. Rev. B 101, 235128 (2020).

[34] J. Park, J. M. Bartlett, H. M. L. Noad, A. Stern, M. E. Barber, M. König, S. Hosoi, T. Shibauchi, A. P. Mackenzie, A. Steppke, and C. W. Hicks, Rigid Platform for Applying Large Tunable Strains to Mechanically Delicate Samples, Rev. Sci. Instrum. 91, 083902 (2020).

[35] T. Noji, T. Suzuki, H. Abe, T. Adachi, M. Kato, and Y. Koike, Growth, Annealing Effects on Superconducting and Magnetic Properties, and Anisotropy of $\mathrm{FeSe}_{1-x} \mathrm{Te}_{x}$ $(0.5 \leq x \leq 1)$ Single Crystals, J. Phys. Soc. Jpn. 79, 084711 (2010).

[36] H.-H. Kuo, M. C. Shapiro, S. C. Riggs, and I. R. Fisher, Measurement of the Elastoresistivity Coefficients of the Underdoped Iron Arsenide $\mathrm{Ba}\left(\mathrm{Fe}_{0.975} \mathrm{Co}_{0.025}\right)_{2} \mathrm{As}_{2}$, Phys. Rev. B 88, 085113 (2013).

[37] M. S. Ikeda, T. Worasaran, J. C. Palmstrom, J. A. W. Straquadine, P. Walmsley, and I. R. Fisher, Symmetric and Antisymmetric Strain as Continuous Tuning Parameters for Electronic Nematic Order, Phys. Rev. B 98, 245133 (2018).

[38] U. S. Kaluarachchi, V. Taufour, A. E. Böhmer, M. A. Tanatar, S. L. Bud'ko, V. G. Kogan, R. Prozorov, and P. C. Canfield, Nonmonotonic Pressure Evolution of the Upper Critical Field in Superconducting FeSe, Phys. Rev. B 93, 064503 (2016).

[39] G. A. Zvyagina, T. N. Gaydamak, K. R. Zhekov, I. V. Bilich, V.D. Fil, D. A. Chareev, and A. N. Vasiliev, Acoustic Characteristics of FeSe Single Crystals, Europhys. Lett. 101, 56005 (2013).

[40] K. Kothapalli, A. E. Böhmer, W. T. Jayasekara, B. G. Ueland, P. Das, A. Sapkota, V. Taufour, Y. Xiao, E. Alp, S. L. Bud'ko, P. C. Canfield, A. Kreyssig, and A. I. Goldman, Strong Cooperative Coupling of PressureInduced Magnetic Order and Nematicity in FeSe, Nat. Commun. 7, 12728 (2016).

[41] S. Margadonna, Y. Takabayashi, M. T. McDonald, K. Kasperkiewicz, Y. Mizuguchi, Y. Takano, A. N. Fitch, E. Suard, and K. Prassides, Crystal Structure of the New $\mathrm{FeSe}_{1-x}$ Superconductor, Chem. Commun. 2008, 5607 (2008).

[42] T. M. McQueen, A. J. Williams, P. W. Stephens, J. Tao, Y. Zhu, V. Ksenofontov, F. Casper, C. Felser, and R. J. Cava, Tetragonal-to-Orthorhombic Structural Phase Transition at $90 \mathrm{~K}$ in the Superconductor $\mathrm{Fe}_{1.01} \mathrm{Se}$, Phys. Rev. Lett. 103, 057002 (2009).

[43] B. A. Frandsen, Q.S. Wang, S. Wu, J. Zhao, and R. J. Birgeneau, Quantitative Characterization of Short-Range Orthorhombic Fluctuations in FeSe through Pair Distribution Function Analysis, Phys. Rev. B 100, 020504(R) (2019).

[44] Q. S. Wang, Y. Shen, B. Y. Pan, Y. Q. Hao, M. W. Ma, F. Zhou, P. Steffens, K. Schmalzl, T. R. Forrest, M. Abdel-Hafiez, 
X. J. Chen, D. A. Chareev, A. N. Vasiliev, P. Bourges, Y. Sidis, H. B. Cao, and J. Zhao, Strong Interplay between Stripe Spin Fluctuations, Nematicity and Superconductivity in FeSe, Nat. Mater. 15, 159 (2016).

[45] M. C. Rahn, R. A. Ewings, S. J. Sedlmaier, S. J. Clarke, and A. T. Boothroyd, Strong $(\pi, 0)$ Spin Fluctuations in $\beta$-FeSe Observed by Neutron Spectroscopy, Phys. Rev. B 91, 180501(R) (2015).

[46] J. C. Palmstrom, A. T. Hristov, S. A. Kivelson, J.-H. Chu, and I. R. Fisher, Critical Divergence of the Symmetric $\left(A_{1 g}\right)$ Nonlinear Elastoresistance Near the Nematic Transition in an Iron-Based Superconductor, Phys. Rev. B 96, 205133 (2017).

[47] Z.-Y. Liu, Y.-H. Gu, W. Zhang, D.-L. Gong, W.-L. Zhang, T. Xie, X.-Y. Lu, X.-Y. Ma, X.-T. Zhang, R. Zhang, J. Zhu, C. Ren, L. Shan, X.-Q. Qiu, P.-C. Dai, Y.-F. Yang, H.-Q. Luo, and S.-L. Li, Nematic Quantum Critical Fluctuations in $\mathrm{BaFe}_{2-x} \mathrm{Ni}_{x} \mathrm{As}_{2}$, Phys. Rev. Lett. 117, 157002 (2016).

[48] H.-C. Mao, D.-L. Gong, X.-Y. Ma, H.-Q. Luo, Y.-F. Yang, L. Shan, and S.-L. Li, Nonlinear Uniaxial Pressure Dependence of the Resistivity in $\mathrm{Sr}_{1-x} \mathrm{Ba}_{x} \mathrm{Fe}_{1.97} \mathrm{Ni}_{0.03} \mathrm{As}_{2}$, Chin. Phys. B 27, 087402 (2018).

[49] M. E. Barber, A. S. Gibbs, Y. Maeno, A. P. Mackenzie, and C. W. Hicks, Resistivity in the Vicinity of a van Hove Singularity: $\mathrm{Sr}_{2} \mathrm{RuO}_{4}$ under Uniaxial Pressure, Phys. Rev. Lett. 120, 076602 (2018).

[50] T. Shimojima, Y. Suzuki, T. Sonobe, A. Nakamura, M. Sakano, J. Omachi, K. Yoshioka, M. Kuwata-Gonomaki, K. Ono, H. Kumigashira, A. E. Böhmer, F. Hardy, T. Wolf, C. Meingast, H. v. Löhneysen, H. Ikeda, and K. Ishizaka, Lifting of $x z / y z$ Orbital Degeneracy at the Structural Transition in Detwinned FeSe, Phys. Rev. B 90, 12111 (R) (2014).

[51] K. Nakayama, Y. Miyata, G. N. Phan, T. Sato, Y. Tanabe, T. Urata, K. Tanigaki, and T. Takahashi, Reconstruction of Band Structure Induced by Electronic Nematicity in an FeSe Superconductor, Phys. Rev. Lett. 113, 237001 (2014).

[52] Y. Suzuki, T. Shimojima, T. Sonobe, A. Nakamura, M. Sakano, H. Tsuji, J. Omachi, K. Yoshioka, M. KuwataGonokami, T. Watashige, R. Kobayashi, S. Kasahara, T. Shibauchi, Y. Matsuda, Y. Yamakawa, H. Kontani, and K. Ishizaka, Momentum-Dependent Sign Inversion of Orbital Order in Superconducting FeSe, Phys. Rev. B 92, 205117 (2015).

[53] S. Onari and H. Kontani, In-Plane Anisotropy of Transport Coefficients in Electronic Nematic States: Universal Origin of Nematicity in Fe-Based Superconductors, Phys. Rev. B 96, 094527 (2017).

[54] Q. S. Wang, Y. Shen, B. Y. Pan, X. W. Zhang, K. Ikeuchi, K. Iida, A. D. Christianson, H. C. Walker, D. T. Adroja, M. Abdel-Hafiez, X. J. Chen, D. A. Chareev, A. N. Vasiliev, and J. Zhao, Magnetic Ground State of FeSe, Nat. Commun. 7, 12182 (2016).

[55] T. Chen, Y. Z. Chen, A. Kreisel, X. Y. Lu, A. Schneidewind, Y. M. Qiu, J. T. Park, T. G. Perring, J. R. Stewart, H. B. Cao, R. Zhang, Y. Li, Y. Rong, Y. Wei, B. M. Andersen, P. J. Hirschfeld, C. Broholm, and P. C. Dai, Anisotropic Spin Fluctuations in Detwinned FeSe, Nat. Mater. 18, 709 (2019).
[56] M. Q. He, L. Wang, F. Hardy, L. P. Xu, T. Wolf, P. Adelmann, and C. Meingast, Evidence for Short-Range Magnetic Order in the Nematic Phase of FeSe from Anisotropic In-Plane Magnetostriction and Susceptibility Measurements, Phys. Rev. B 97, 104107 (2018).

[57] M. Breitkreiz, P. M. R. Brydon, and C. Timm, Resistive Anisotropy due to Spin-Fluctuation Scattering in the Nematic Phase of Iron Pnictides, Phys. Rev. B 90, 121104(R) (2014).

[58] C.-C. Chen, J. Maciejko, A. P. Sorini, B. Moritz, R. R. P. Singh, and T. P. Devereaux, Orbital Order and Spontaneous Orthorhombicity in Iron Pnictides, Phys. Rev. B 82, 100504 (R) (2010).

[59] M. Breitkreiz, P. M. R. Brydon, and C. Timm, Transport in Multiband Systems with Hot Spots on the Fermi Surface: Forward-Scattering Corrections, Phys. Rev. B 89, 245106 (2014).

[60] M. Schütt, J. Schmalian, and R. M. Fernandes, Origin of $d c$ and ac Conductivity Anisotropy in Iron-Based Superconductors: Scattering Rate versus Spectral Weight Effects, Phys. Rev. B 94, 075111 (2016).

[61] R. Fernández-Martín, L. Fanfarillo, L. Benfatto, and B. Valenzuela, Anisotropy of the dc Conductivity due to Orbital-Selective Spin Fluctuations in the Nematic Phase of Iron Superconductors, Phys. Rev. B 99, 155117 (2019).

[62] M. Chinotti, A. Pal, L. Degiorgi, A. E. Böhmer, and P. C. Canfield, Ingredients for the Electronic Nematic Phase in FeSe Revealed by Its Anisotropic Optical Response, Phys. Rev. B 98, 094506 (2018).

[63] R. M. Fernandes, E. Abrahams, and J. Schmalian, Anisotropic In-Plane Resistivity in the Nematic Phase of the Iron Pnictides, Phys. Rev. Lett. 107, 217002 (2011).

[64] D. Gugan, The Electrical Resistivity of Potassium below 4.2 K, Proc. R. Soc. A 325, 223 (1971).

[65] C. W. Hicks, A. S. Gibbs, A. P. Mackenzie, H. Takatsu, Y. Maeno, and E. A. Yelland, Quantum Oscillations and High Carrier Mobility in the Delafossite $\mathrm{PdCoO}_{2}$, Phys. Rev. Lett. 109, 116401 (2012).

[66] Y. Yamakawa, S. Onari, and H. Kontani, Nematicity and Magnetism in FeSe and Other Families of Fe-Based Superconductors, Phys. Rev. X 6, 021032 (2016).

[67] A. V. Chubukov, R. M. Fernandes, and J. Schmalian, Origin of Nematic Order in FeSe, Phys. Rev. B 91, 201105(R) (2015).

[68] A. V. Chubukov, M. Khodas, and R. M. Fernandes, Magnetism, Superconductivity, and Spontaneous Orbital Order in Iron-Based Superconductors: Which Comes First and Why?, Phys. Rev. X 6, 041045 (2016).

[69] Max Planck Digital Library, https://edmond.mpdl.mpg.de/ imeji/collection/4O6T_XGGtgnRDFd.

[70] M. Ghini, M. Bristow, J. C. A. Prentice, S. Sutherland, S. Sanna, A. A. Haghighirad, and A. I. Coldea, companion paper, Strain Tuning of Nematicity and Superconductivity in Single Crystals of FeSe, Phys. Rev. B 103, 205139 (2021).

[71] M. C. Shapiro, A. T. Hristov, J. C. Palmstrom, Jiun-Haw $\mathrm{Chu}$, and I. R. Fisher, Measurement of the $B_{1 g}$ and $B_{2 g}$ Components of the Elastoresistivity Tensor for Tetragonal Materials via Transverse Resistivity Configurations, Rev. Sci. Instrum. 87, 063902 (2016).

[72] H. C. Montgomery, Method for Measuring Electrical Resistivity of Anisotropic Materials, J. Appl. Phys. 42, 2971 (1971). 
[73] J. D. Wasscher, Note on Four-Point Resistivity Measurements on Anisotropic Conductors, Philips Res. Rep. 16, 301 (1961), https://ci.nii.ac.jp/naid/10009582567/en/.

[74] C. A. M. dos Santos, A. de Campos, M. S. da Luz, B. D. White, J. J. Neumeier, B.S. de Lima, and C. Y. Shigue, Procedure for Measuring Electrical Resistivity of Anisotropic Materials: A Revision of the Montgomery Method, J. Appl. Phys. 110, 083703 (2011).

[75] L. J. Van der Pauw, Determination of Resistivity Tensor and Hall Tensor of Anisotropic Shape, Philips Res. Rep. 16, 187 (1961).

[76] C. W. Hicks, M. E. Barber, S. D. Edkins, D. O. Brodsky, and A. P. Mackenzie, Piezoelectric-Based Apparatus for Strain Tuning, Rev. Sci. Instrum. 85, 065003 (2014).
[77] J. N. Millican, D. Phelan, E. L. Thomas, J. B. Leão, and E. Carpenter, Pressure-Induced Effects on the Structure of the FeSe Superconductor, Solid State Commun. 149, 707 (2009).

[78] S. Margadonna, Y. Takabayashi, Y. Ohishi, Y. Mizuguchi, Y. Takano, T. Kagayama, T. Nakagawa, M. Takata, and K. Prassides, Pressure Evolution of the Low-Temperature Crystal Structure and Bonding of the Superconductor FeSe ( $\left.T_{c}=37 \mathrm{~K}\right)$, Phys. Rev. B 80, 064506 (2009).

[79] T. Hashimoto and A. Ikushima, Mechanical Properties of Stycast-1266 at Low Temperatures, Rev. Sci. Instrum. 51, 378 (1980).

[80] S. Chandra and A. K. M. A. Islam, Elastic Properties of Monoand Poly-Crystalline PbO-Type $\mathrm{FeSe}_{1 x} \mathrm{Te}_{x}(x=0-1.0)$ : A First-Principles Study, Physica (Amsterdam) 470C, 2072 (2010). 